Revue des patrimoines

\title{
Réhabilitation, gestion et mise en valeur touristique de l'enceinte urbaine d'Ibiza (Espagne)
}

Fernando Cobos-Guerra

\section{(2) OpenEdition}

Journals

Édition électronique

URL : http://journals.openedition.org/insitu/792

DOI : 10.4000/insitu.792

ISSN : 1630-7305

Éditeur

Ministère de la culture

Référence électronique

Fernando Cobos-Guerra, «Réhabilitation, gestion et mise en valeur touristique de l'enceinte urbaine d'Ibiza (Espagne) », In Situ [En ligne], 16 | 2011, mis en ligne le 26 juillet 2011, consulté le 30 avril 2019. URL : http://journals.openedition.org/insitu/792 ; DOI : 10.4000/insitu.792

Ce document a été généré automatiquement le 30 avril 2019

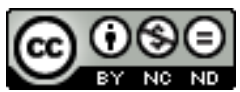

In Situ Revues des patrimoines est mis à disposition selon les termes de la licence Creative Commons Attribution - Pas d'Utilisation Commerciale - Pas de Modification 4.0 International. 


\section{Réhabilitation, gestion et mise en valeur touristique de l'enceinte urbaine d'Ibiza (Espagne)}

Fernando Cobos-Guerra

[Traduit de l'espagnol par Dominique Blanc]

La Muraille et le Plan Directeur 


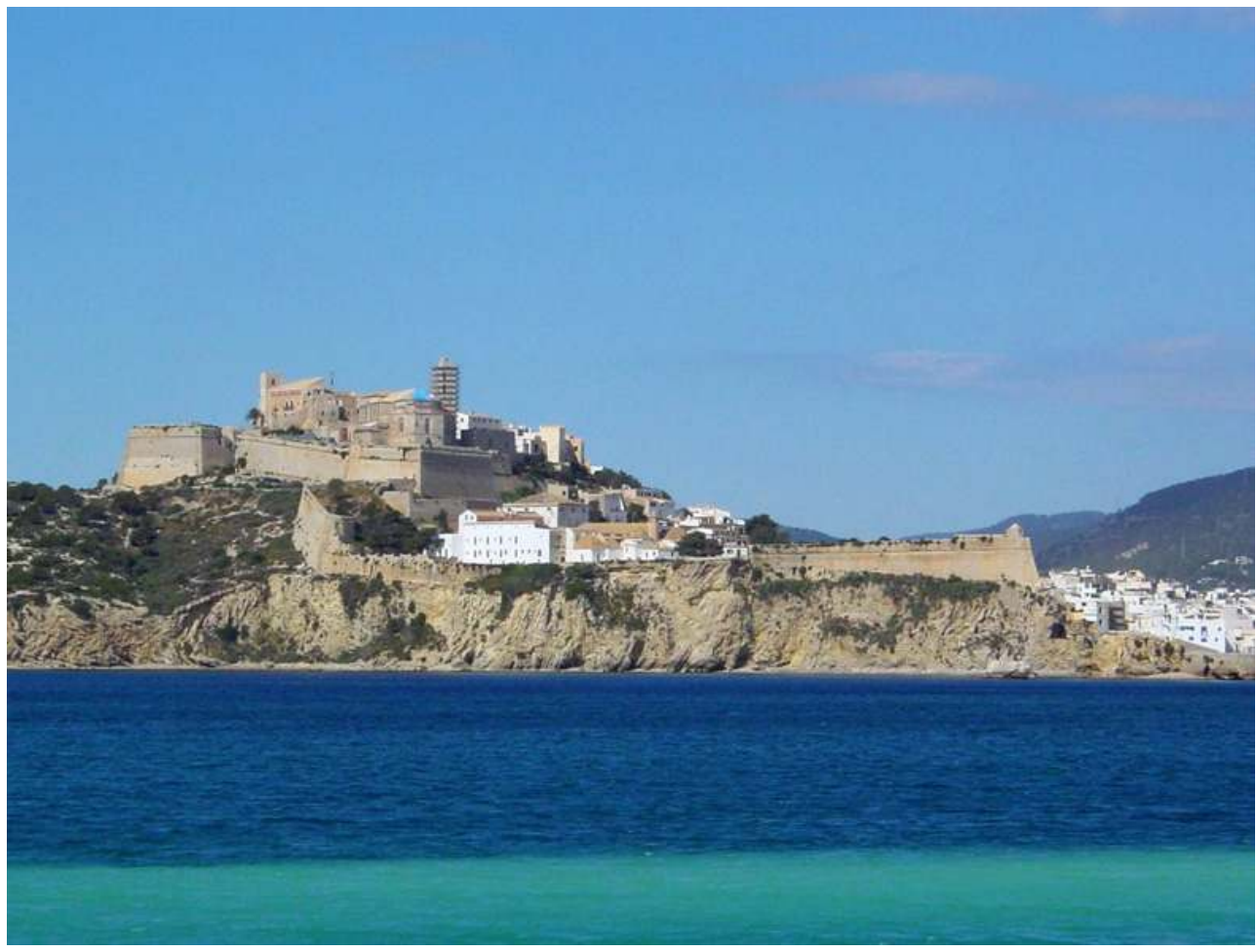

Dalt Vila (la Ville Haute) et son enceinte, vues de la mer.

(c) F. Cobos.

2 L'enceinte d'Ibiza, construite à la Renaissance est l'exemplaire le mieux conservé en Europe d'un prototype de fortification qui définit, développa et fut un élément clé de l'expansion culturelle et politique de l'Europe de la Renaissance au-delà de ses limites géographiques. En effet, à l'exception des fortifications réalisées en Italie par Sangallo et le Valencien Pedro Luis Escrivá (L'Aquila ou San Telmo de Naples), les œuvres de la première période de la Renaissance qui n'ont pas été modifiées aux siècles suivants sont peu nombreuses. En 1942, l'enceinte fut déclarée Monument National pour son intérêt paysager, et en 1969 tout le quartier de Dalt Vila (la Ville Haute) fut reconnu comme Ensemble Historique grâce à l'exceptionnelle conservation de son tissu urbain. L'inscription au Patrimoine Mondial en 1999 s'est appuyée sur sa condition d'élément exceptionnel dans l'histoire de la technologie du modèle de fortification hispanoitalienne qu'il fallait désormais comprendre en fonction des règles de construction propres aux fortifications bastionnées en les replaçant dans l'univers culturel et scientifique de la Renaissance. 


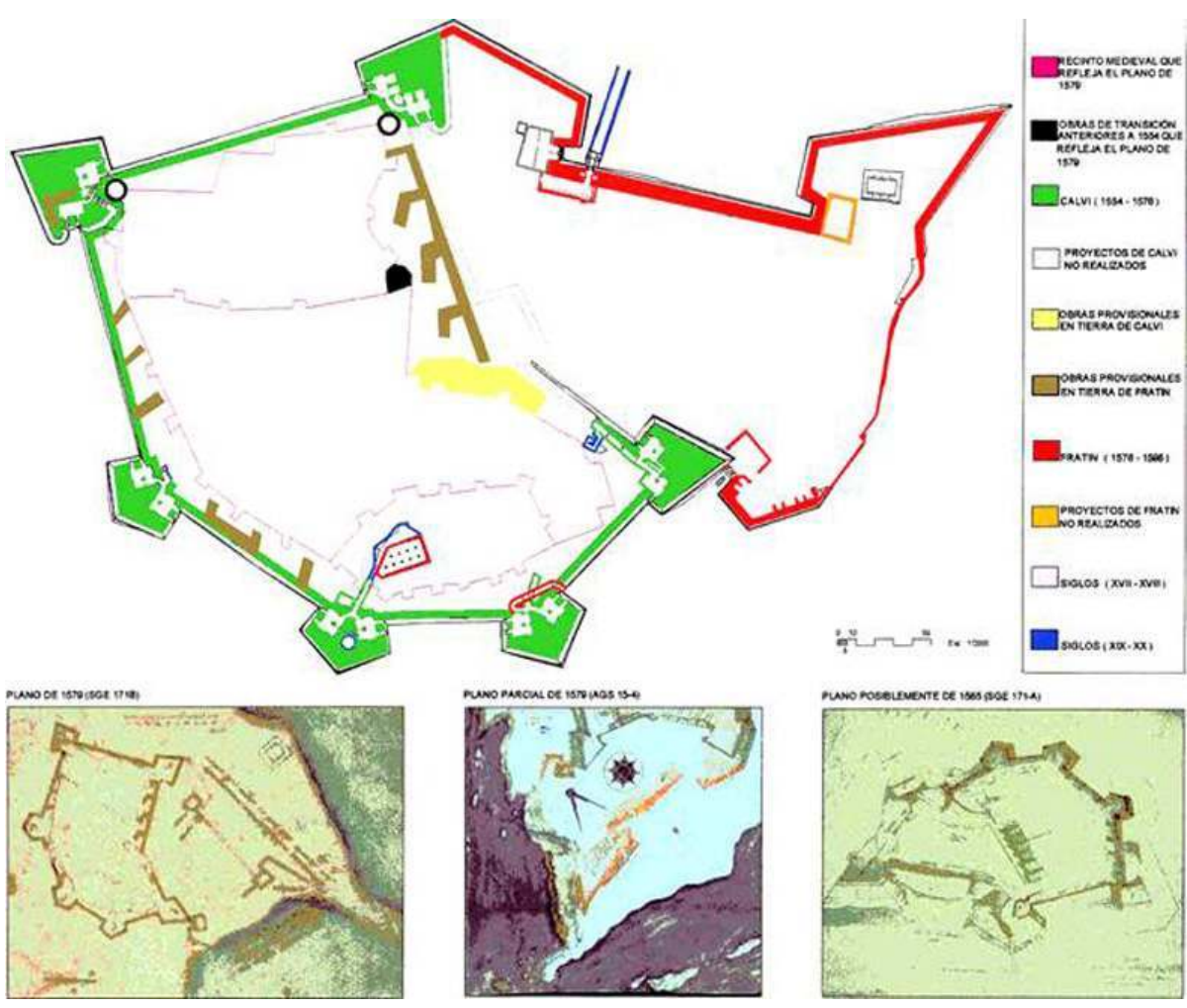

Étapes de la construction. En vert les fortifications dessinées par Calvi à partir de 1554. En rouge les fortifications dessinées par Fratín à partir de 1576.

(c) F. Cobos, cabinet du plan directeur.

Le premier traité de fortification écrit en castillan, l'« Apología » de Pedro Luis Escrivá (Naples, 1538) cite Vitruve pour qui «l'architecture doit être une musique bien accordée »; les ingénieurs du XVI étaient bien conscients du fait que la fortification de la Renaissance est le résultat d'un système géométrique, et donc mathématique, dans lequel tous les éléments sont en relation et se répondent les uns aux autres suivant des règles de composition mathématiques, optiques et géométriques très précises. Ce qui revient à dire qu'il existe une harmonie entre toutes les parties qui leur permet de "résonner» ensemble de manière accordée. 


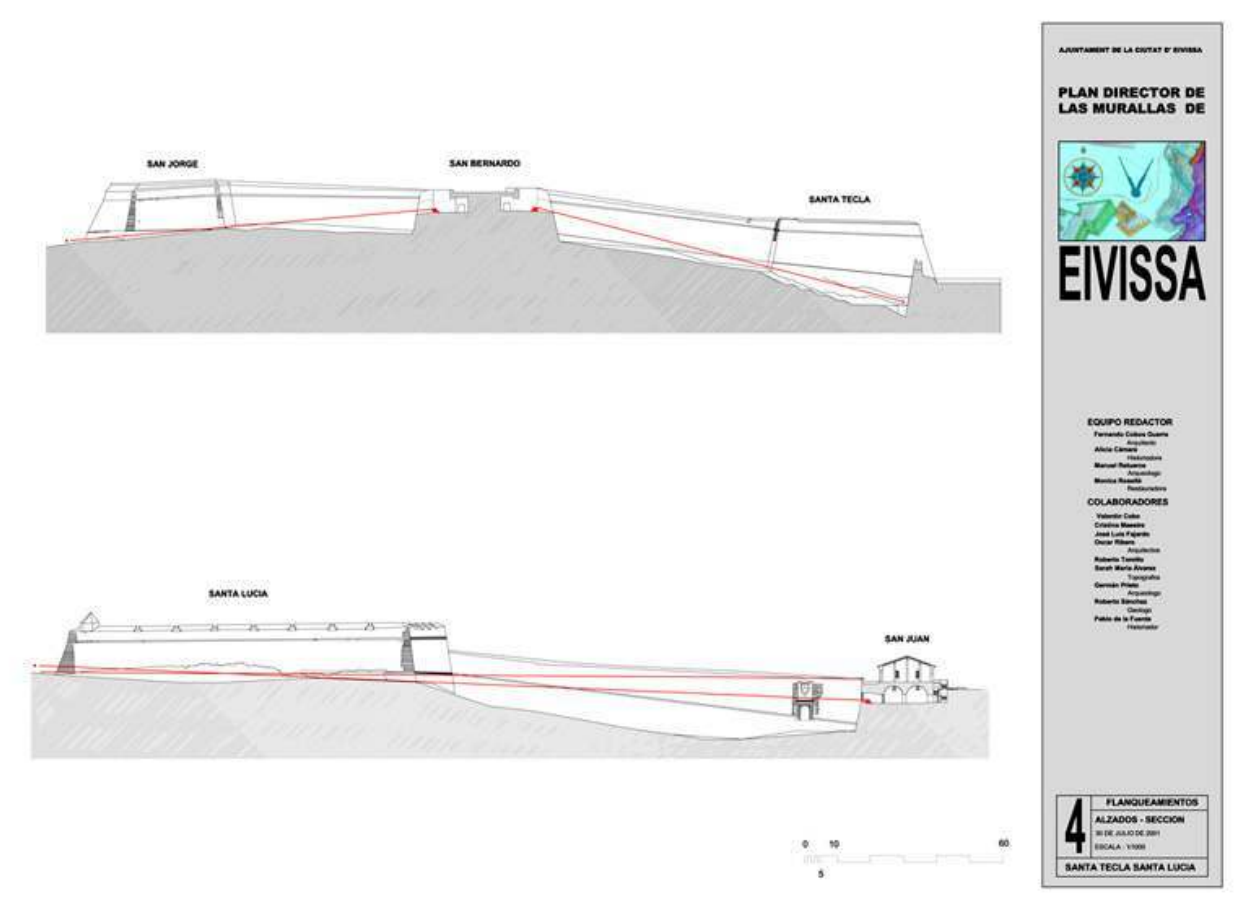

Étude de la ligne rasante extérieure de la muraille établie à partir des bouches à feu des casemates.

(c) F. Cobos, cabinet du plan directeur.

4 Le tracé de la Renaissance, saisi non seulement à travers le plan mais aussi les lignes rasantes, la disposition des casemates, l'ouverture des meurtrières, l'épaisseur des parapets, la taille de la pierre..., devenait ainsi une valeur déterminante et propre à cette fortification si singulière. Analyser - et expliquer - cela, pour pouvoir intervenir ensuite avec rigueur et discernement, telle était l'idée de départ des rédacteurs du Plan Directeur des Murailles Renaissance quand, au début de l'année 2001, nous avons commencé notre travail à Ibiza. La lecture pluridisciplinaire du monument réalisée alors - « comprendre la construction en lisant les documents et comprendre les documents en lisant la construction » - a déterminé (comme nous le verrons) l'ensemble des propositions, des choix et des interventions d'un Plan Directeur qui fut récompensé lors de l'attribution des Prix Européens du Patrimoine (EUROPA NOSTRA) en 2004 et dont l'étude historique et architecturale fut publiée en 2008 sous le titre De la fortificación d'Yviça. 
Figure 4

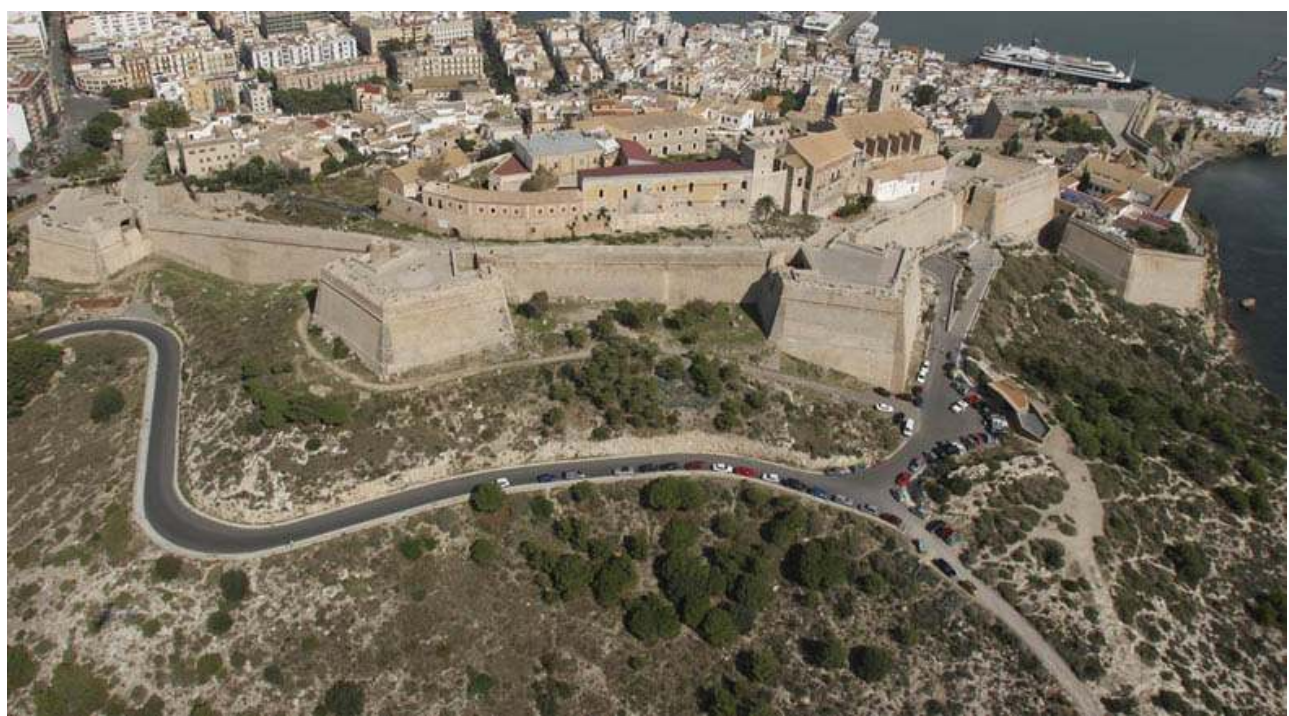

La muraille faisant face au Puig de Los Molinos (la partie historiquement la plus vulnérable de la Cité d'lbiza).

(c) Mairie d'Ibiza, cabinet du plan directeur.

5 Sans négliger la préservation des valeurs reconnues du monument comme son image paysagère, son environnement urbain ou sa complexité stratigraphique, l'objectif le plus novateur du plan était «la récupération des significations et des éléments clé de la muraille en suivant la méthodologie et les caractéristiques propres aux enceintes bastionnées ». Il s'agissait d'établir comme critère la préservation et la mise en valeur des caractéristiques techniques formelles et fonctionnelles du projet de la Renaissance en tant qu'organisme complexe mais unitaire régi par des règles propres à sa condition de fortification bastionnée.

\section{Premières interventions : casemates et bastions}


Figure 5

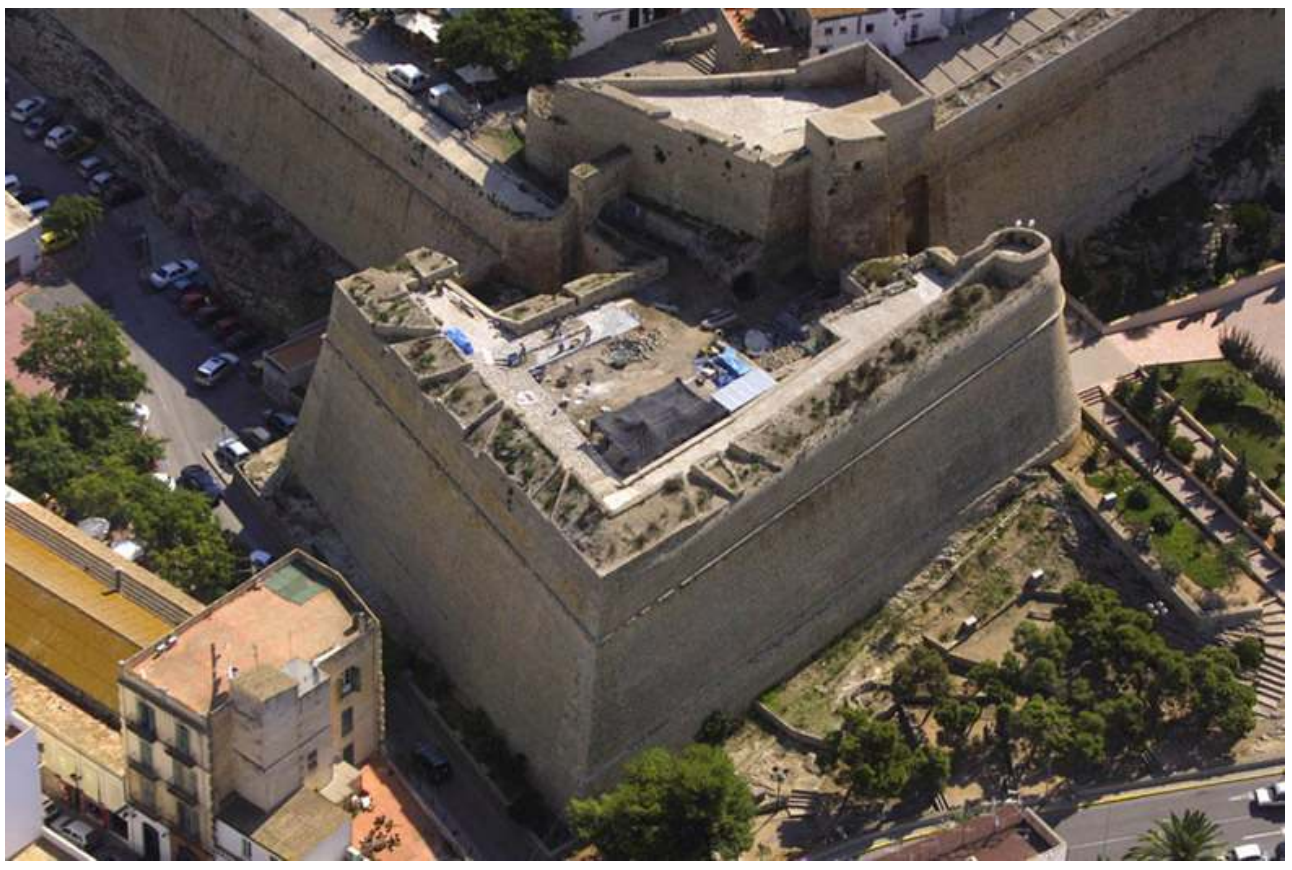

Bastion de San Pedro (Sant Pere) pendant l'excavation de la plate-forme.

(c) Mairie d'Ibiza, cabinet du plan directeur.

\section{Figure 6}
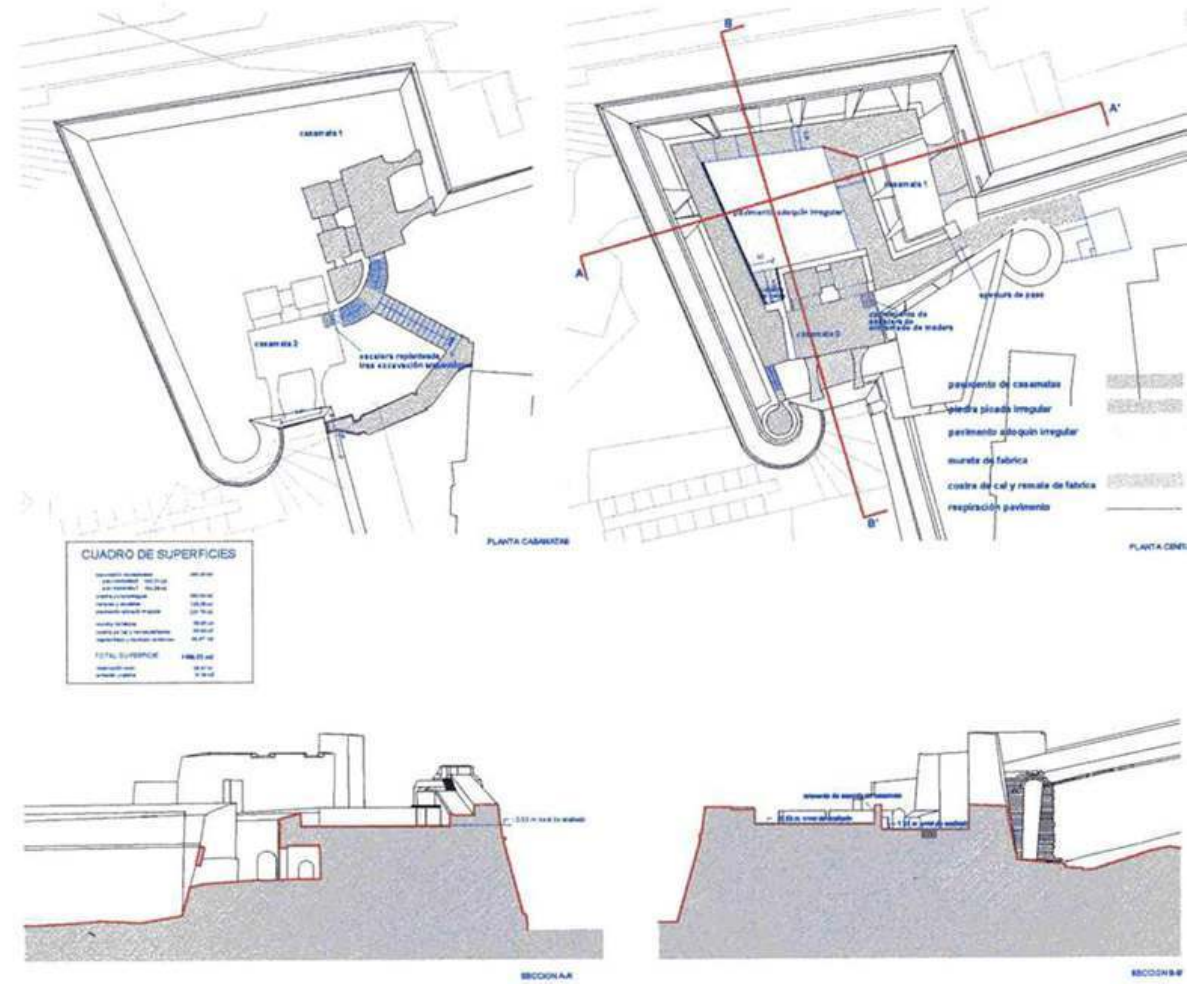

Projet de restauration des casemates et de la plate-forme du bastion de San Pedro.

(c) F. Cobos, maîtrise d'œuvre du plan directeur. 
Étant entendu que la fortification est définie essentiellement en plan et en coupe par le feu croisé des casemates, que ce n'est qu'en partant de ces dernières que l'on peut comprendre réellement comment fonctionne cette enceinte et que, de plus, ces espaces sont les plus beaux de la muraille avec leurs voûtes et leurs tunnels, cela n'avait pas de sens qu'elles restent inaccessibles, qu'elles soient occupées par des réserves d'eau obsolètes ou défigurées par des bains publics ou des restes d'anciennes discothèques. Pour cette raison, l'un des premiers objectifs (en grande partie déjà atteint) fut de les réhabiliter, de les rendre visitables et d'en profiter pour expliquer l'enceinte aux visiteurs, en partant des casemates, grâce à une muséification attractive documentée par les études du Plan Directeur, et, plus important encore, pour l'expliquer aux habitants d'Ibiza qui n'avaient jamais pu accéder à ces lieux.

\section{Figure 7}

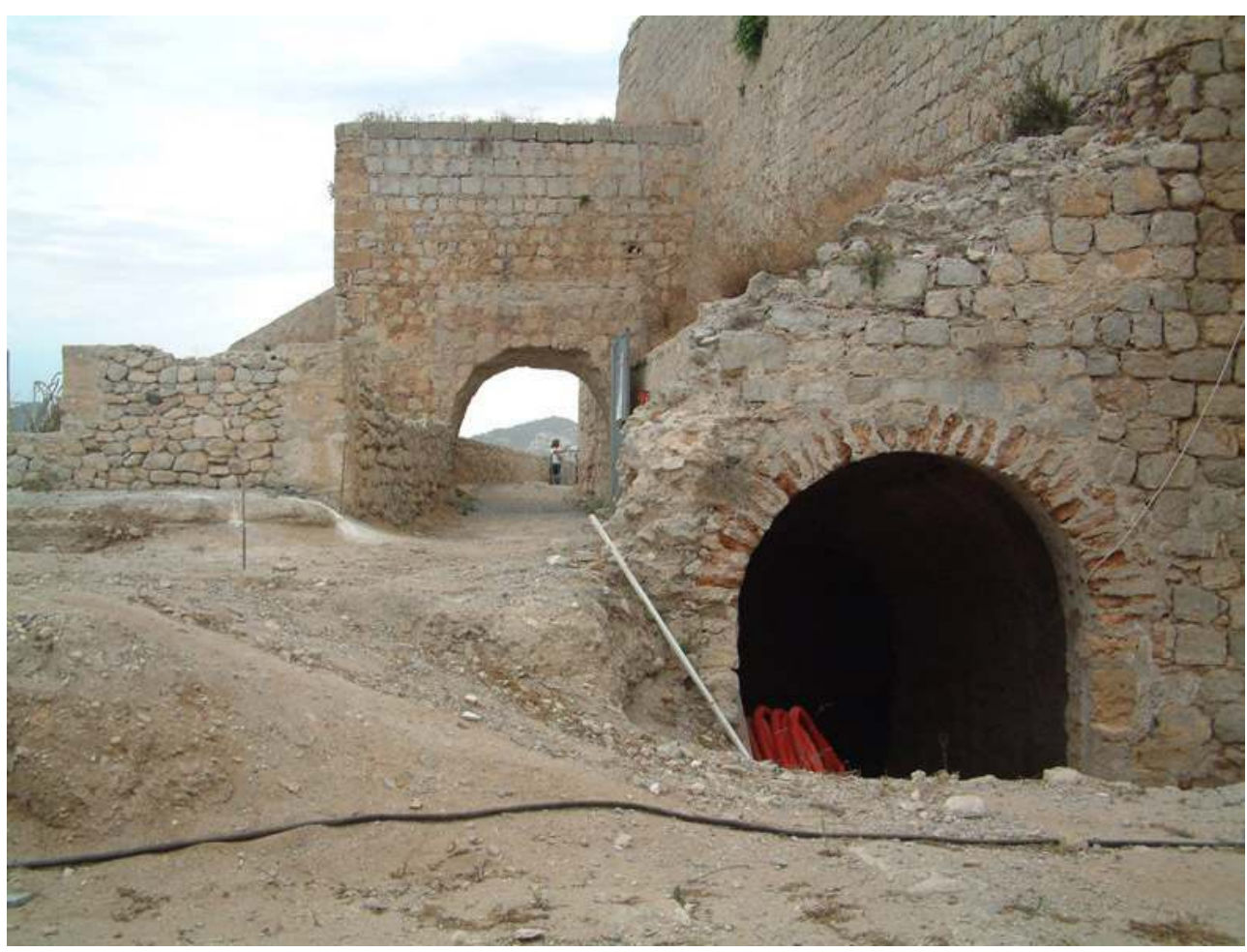

Excavation de la plate-forme de San Pedro.

(C) F. Cobos, maîtrise d'œuvre du plan directeur. 
Figure 8

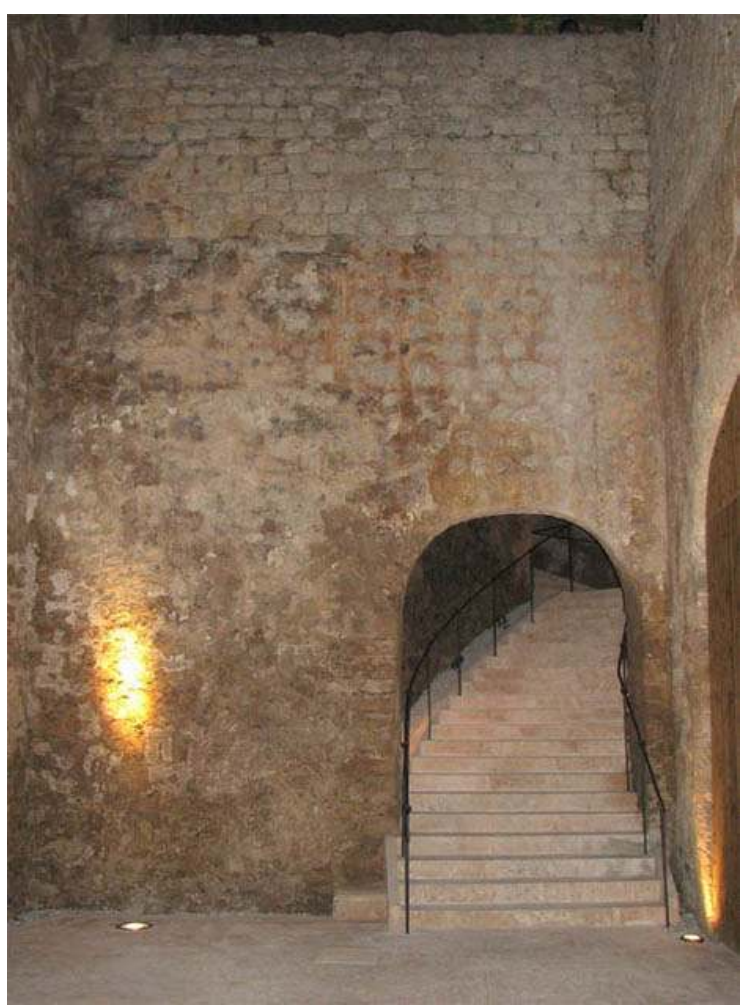

Tunnels de descente à la casemate inférieure de San Pedro. (C) F. Cobos, maîtrise d'œuvre du plan directeur.

\section{Figure 9}

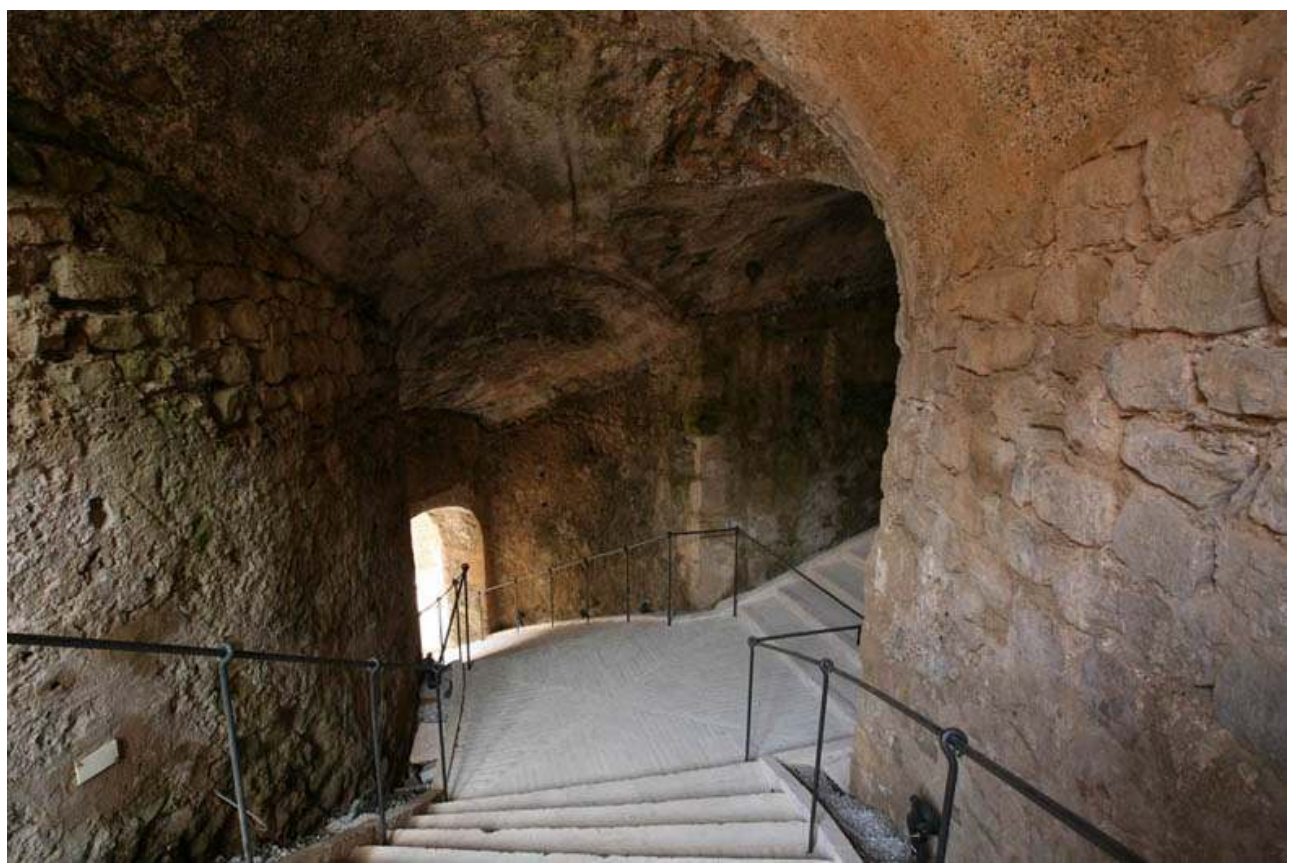

Tunnels de descente à la casemate inférieure de San Pedro.

(c) F. Cobos, maîtrise d'œuvre du plan directeur. 
Figure 10

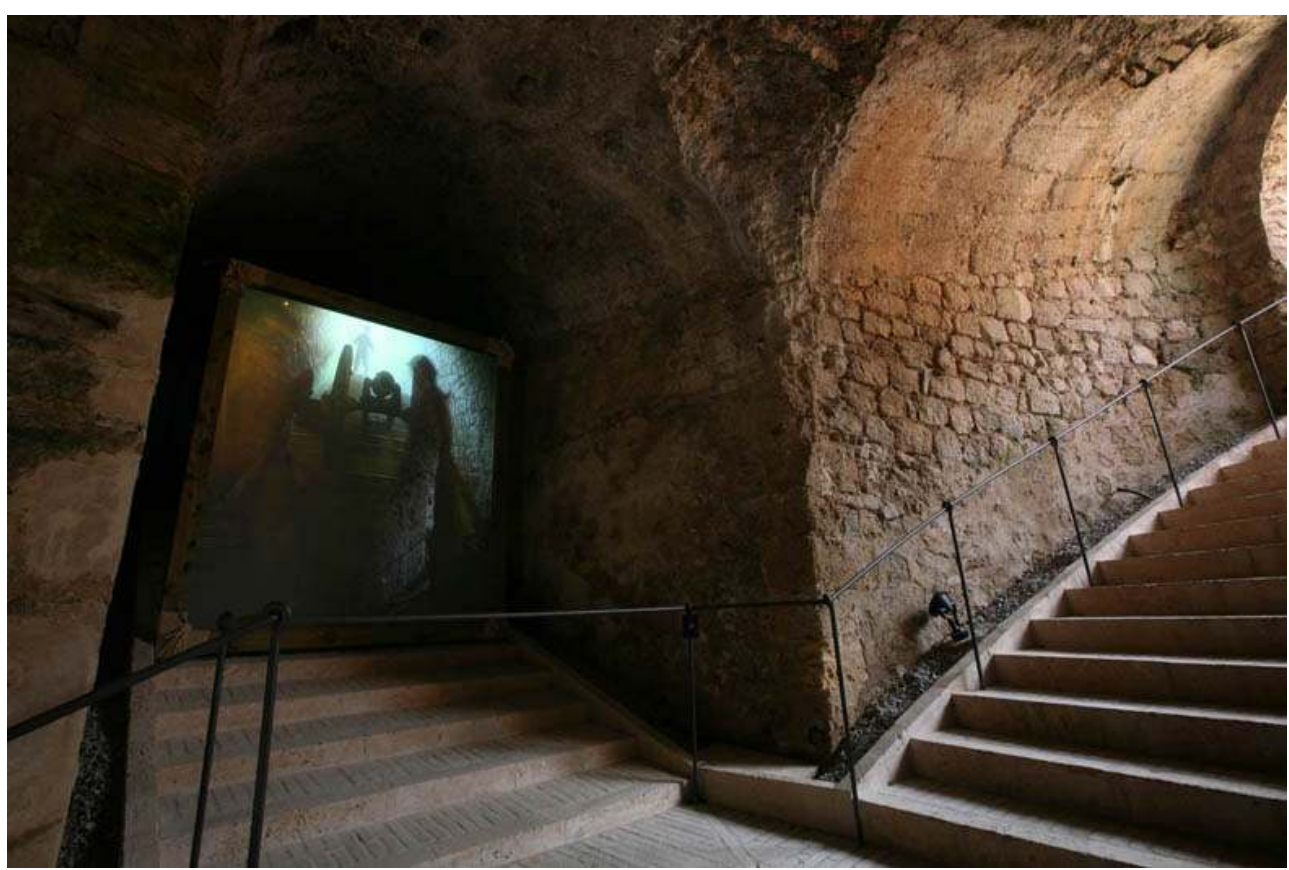

Tunnels et casemates de San Pedro déjà muséifiées.

(c) Mairie d'Ibiza, maîtrise d'œuvre du plan directeur.

7 Parmi tous les bastions et casemates réhabilités, l'action la plus importante a été menée à bien au bastion de San Pedro qui présentait l'intérêt d'être resté inachevé à cause d'une succession d'infortunes qui débutèrent en 1562 avec le naufrage où périt le maître d'œuvre Antonio Jaime de retour de Perpignan où il avait réceptionné les plans dessinés par Calvi pour ce bastion. Transformé à un point tel qu'il fut planté d'arbres et hébergea une ancienne discothèque fermée voici trente ans, il subit une intervention qui consista à excaver la plate-forme jusqu'à ce que soit retrouvée le tracé de la casemate supérieure inachevée, à démolir les restes de la discothèque, à réparer les profonds dommages causés aux parements, à retrouver et rouvrir les accès, escaliers et tunnels, et à réparer la casemate inférieure en vue de sa muséification. 


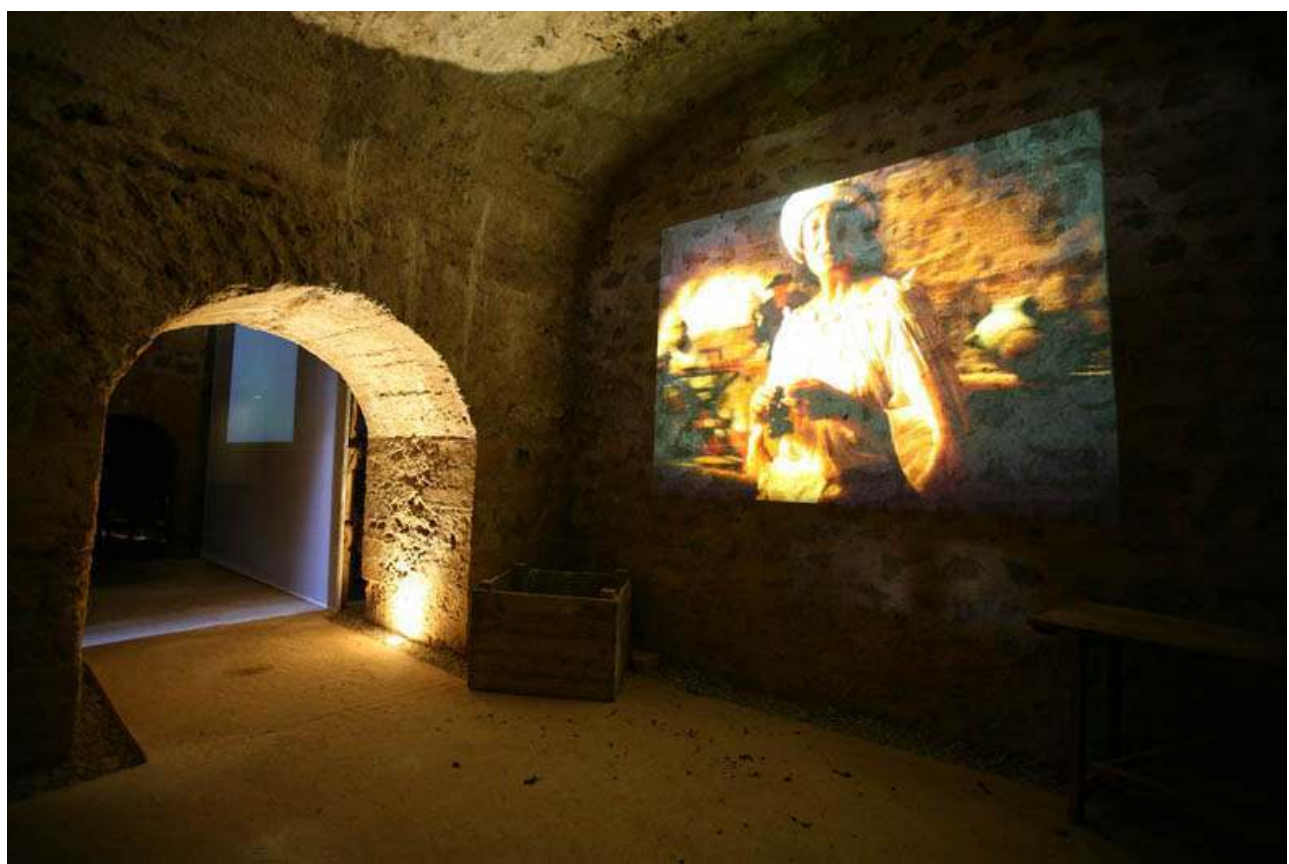

Tunnels et casemates de San Pedro déjà muséifiées.

(c) Mairie d'Ibiza, maîtrise d'œuvre du plan directeur.

\section{Les parapets}

Figure 12

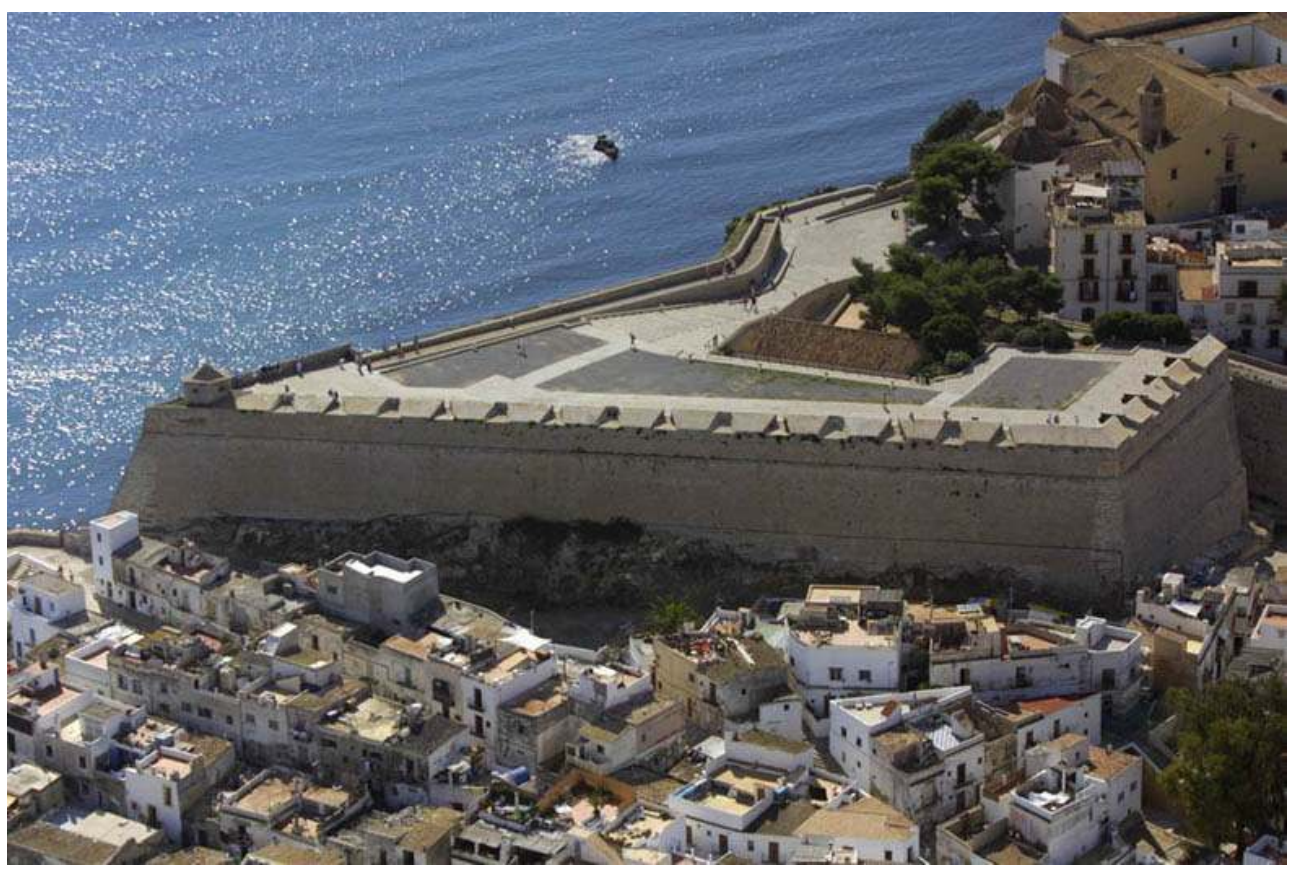

Parapets de Santa Lucía avec leur crépi de chaux reconstitué.

(c) Mairie d'Ibiza, cabinet du plan directeur. 
Figure 13

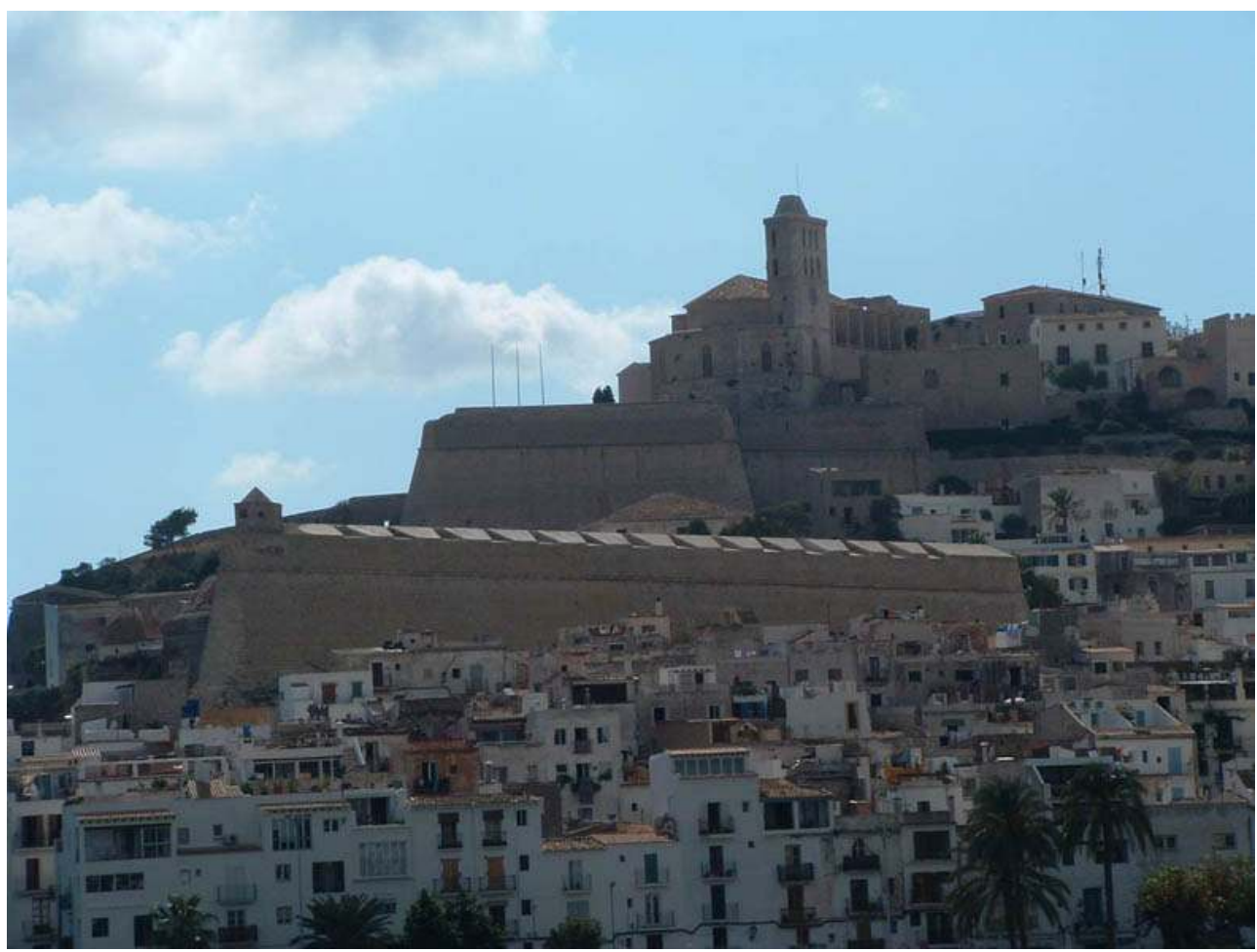

Parapets de Santa Lucía avec leur crépi de chaux reconstitué.

(c) Mairie d'Ibiza, cabinet du plan directeur.

8 Un autre élément important du Plan consista à caractériser l'évolution des parapets de la muraille. Trois types principaux furent détectés ainsi qu'une infinité de variantes de ces types principaux, ce qui signifiait pratiquement que chaque tronçon de la muraille présentait une finition différente, fruit d'une histoire différente et d'une solution de restauration différente, et parfois pas très heureuse. À partir de là, connaître l'histoire particulière de chaque tronçon de muraille devint la condition essentielle pour les travaux de consolidation et de finition des parapets, indispensables pour éviter les éboulements, les infiltrations dans le mur et sa colonisation par les indestructibles câpriers qui revendiquent avec entêtement leur place depuis que la muraille est venue occuper l'espace de leurs anciens jardins il y a presque cinq siècles. 


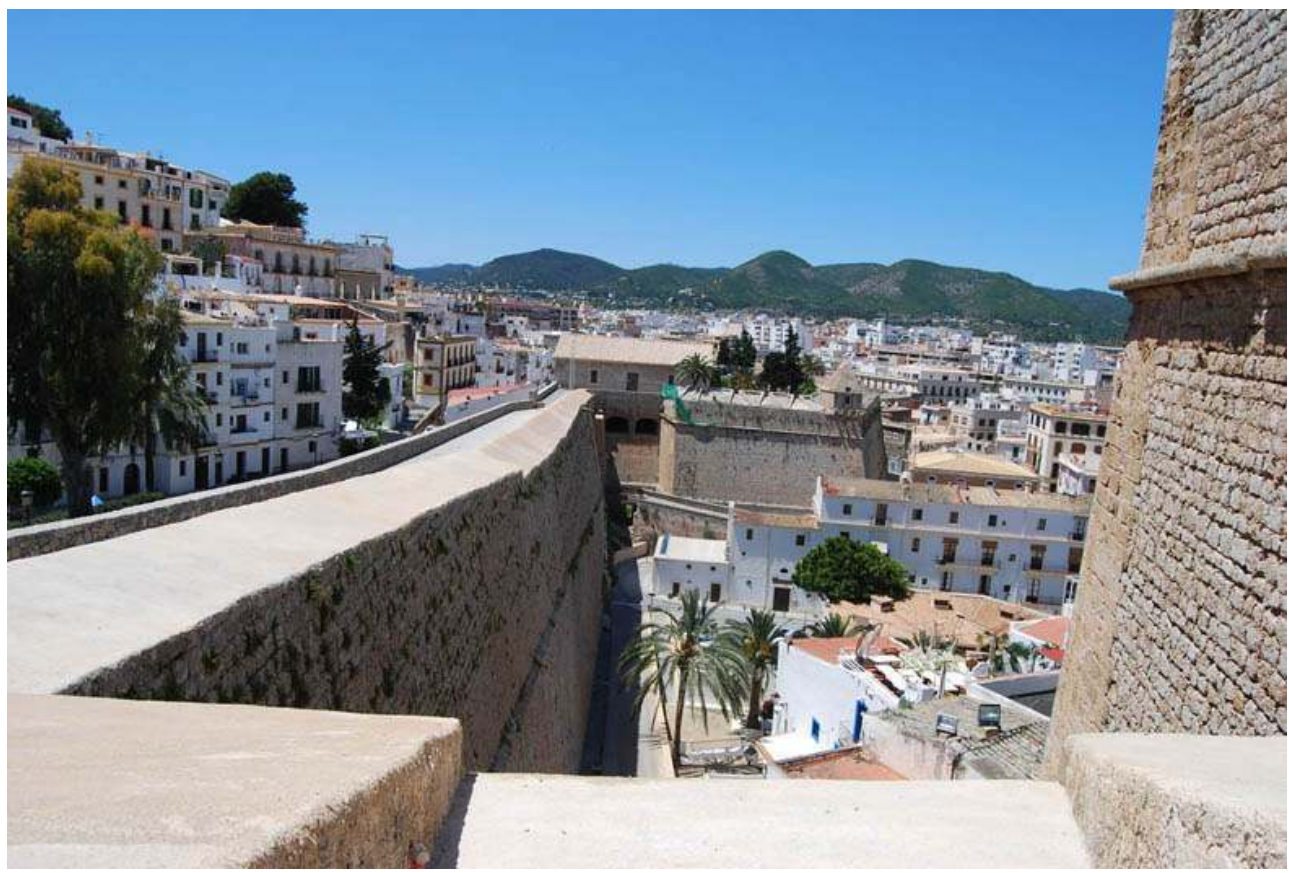

Couronnement de la courtine partiellement inachevée entre les bastions de Santa Lucía et de San Juan

(c) F. Cobos, maîtrise d'œuvre du plan directeur.

\section{Figure 15}

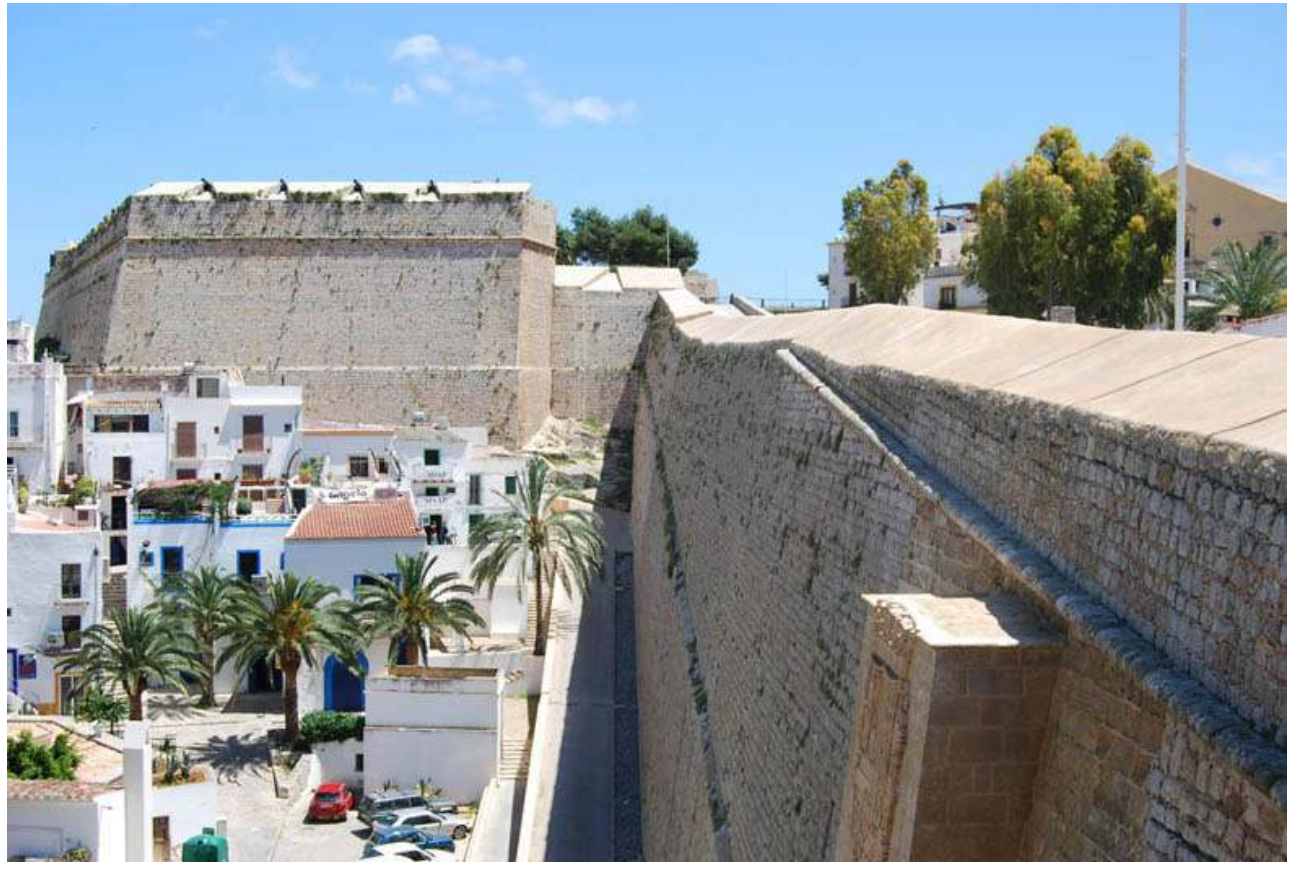

Couronnement de la courtine partiellement inachevée entre les bastions de Santa Lucía et de San Juan.

(c) F. Cobos, maîtrise d'œuvre du plan directeur. 


\section{Les portes}

Figure 16

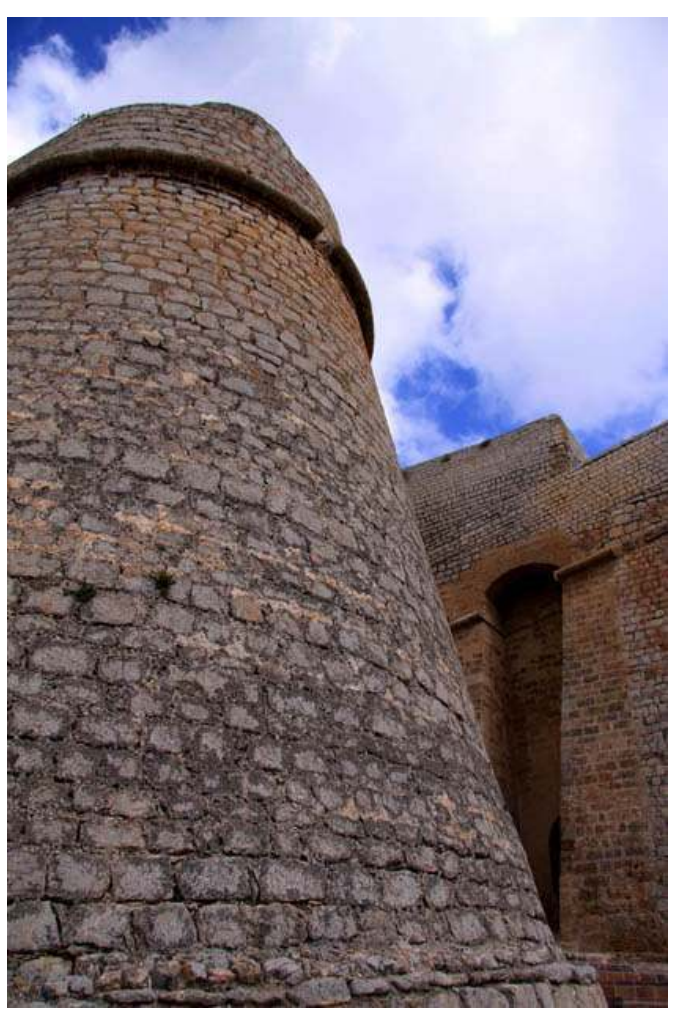

Le Portal Nou (Porte Neuve) occulté et protégé par l'orillon du bastion de San Pedro.

(c) F. Cobos, maîtrise d'œuvre du plan directeur. 
Figure 17

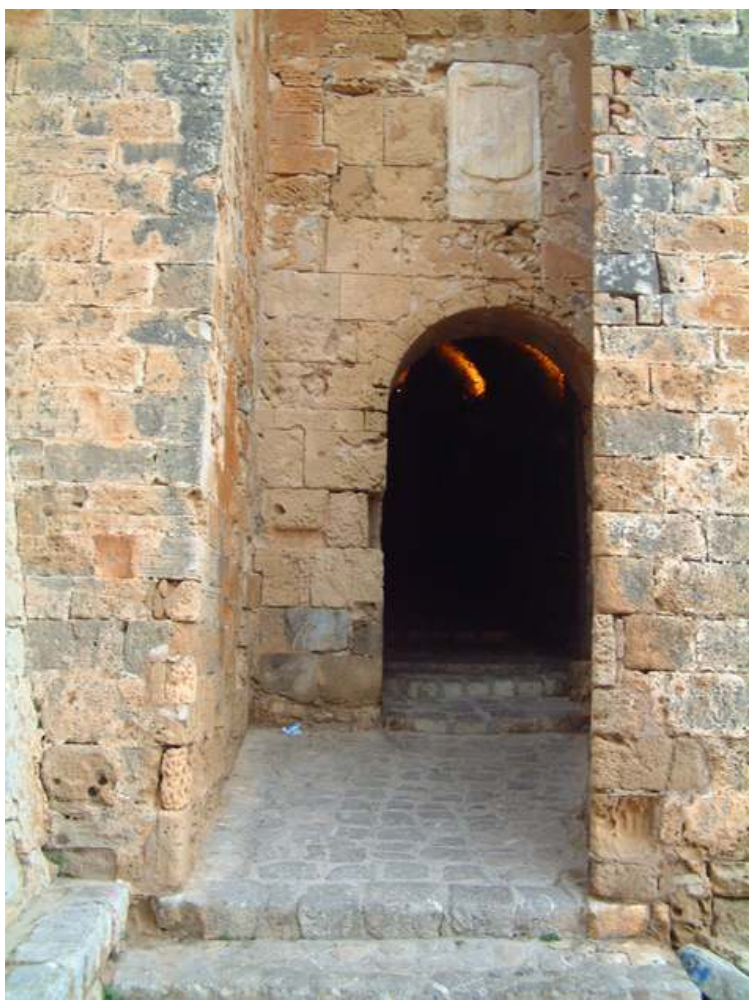

Récupération de la stéréotomie originelle du Portal Nou où l'on observe le stade initial avec le placage de $12 \mathrm{~cm}$ du XIXe siècle, étude de la stéréotomie originelle de l'arc à partir des pièces de la

Renaissance qui se trouvaient derrière le placage, en partie mutilées, et réhabilitation de l'arc et de la stéréotomie originelle.

(c) F. Cobos, maîtrise d'œuvre du plan directeur. 
Figure 18

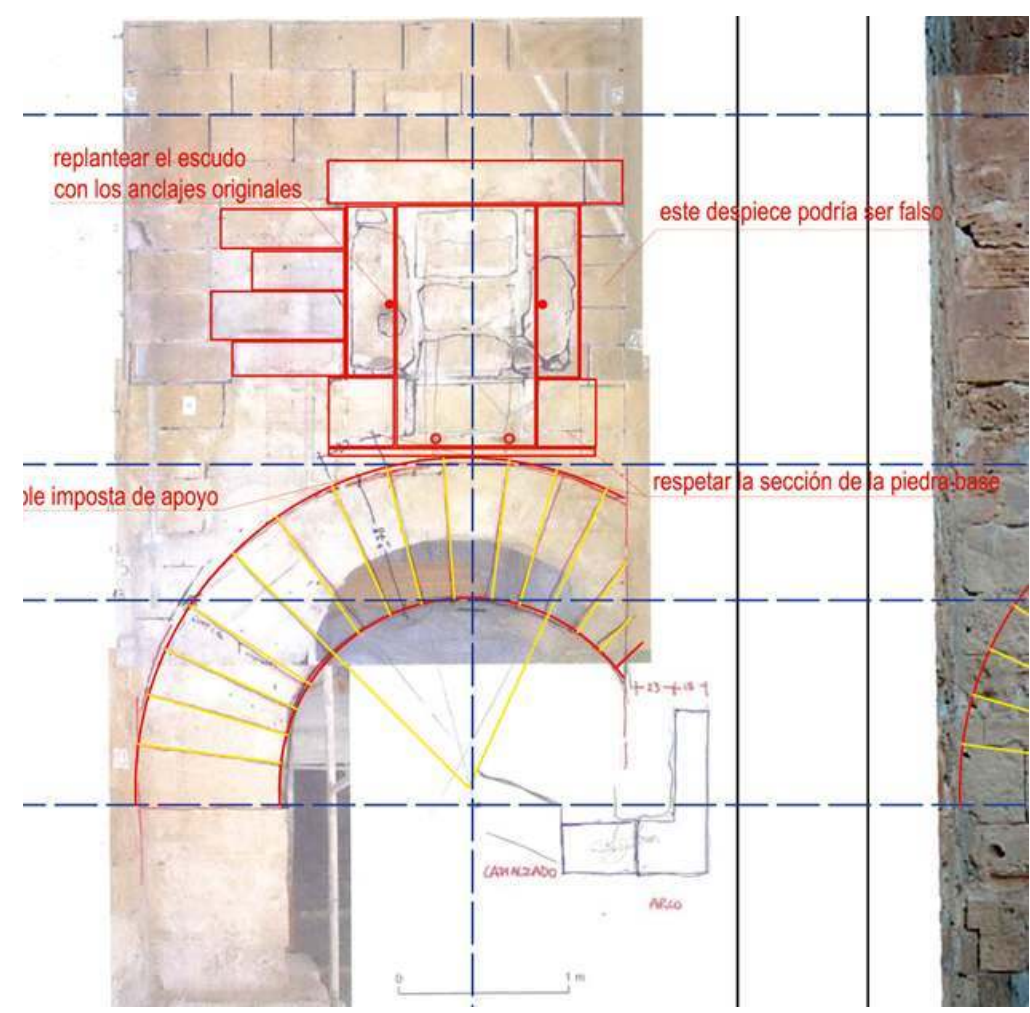

Récupération de la stéréotomie originelle du Portal Nou où l'on observe le stade initial avec le placage de $12 \mathrm{~cm}$ du XIXe siècle, étude de la stéréotomie originelle de l'arc à partir des pièces de la

Renaissance qui se trouvaient derrière le placage, en partie mutilées, et réhabilitation de l'arc et de la stéréotomie originelle.

(c) F. Cobos, maîtrise d'œuvre du plan directeur. 
Figure 19

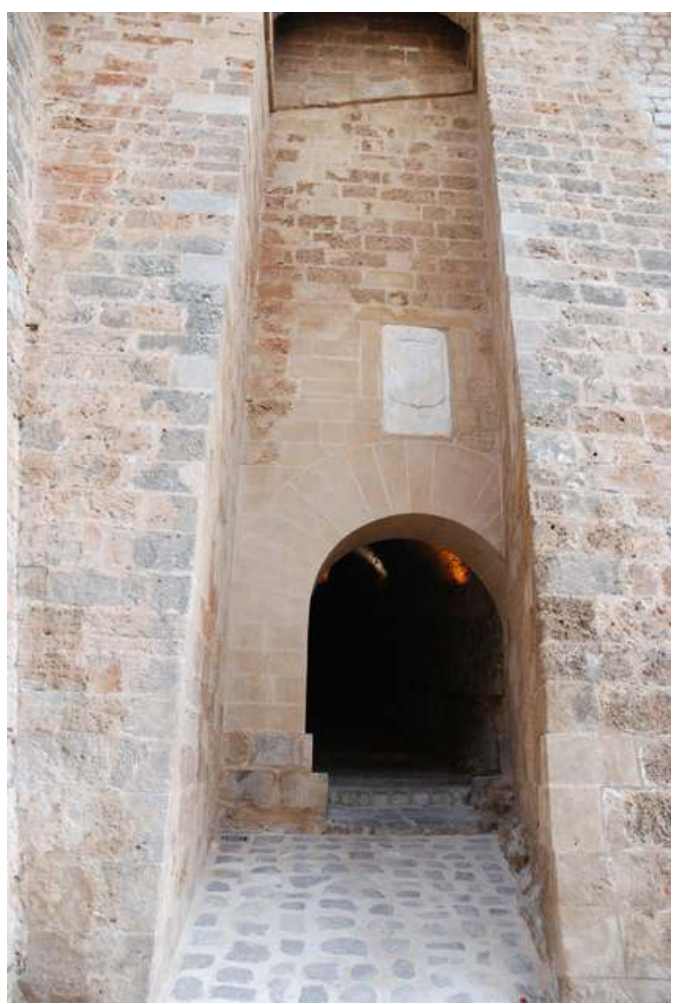

Récupération de la stéréotomie originelle du Portal Nou où l'on observe le stade initial avec le placage de $12 \mathrm{~cm}$ du XIX ${ }^{e}$ siècle, étude de la stéréotomie originelle de l'arc à partir des pièces de la

Renaissance qui se trouvaient derrière le placage, en partie mutilées, et réhabilitation de l'arc et de la stéréotomie originelle.

(c) F. Cobos, maîtrise d'œuvre du plan directeur.

$9 \quad$ Les recherches documentaires ont permis de comprendre que l'aspect inégal des pierres de Marès de la muraille (ainsi que leurs différents degrés de détérioration et leur pathologie actuelle) était lié à leur diversité de provenance; le changement de lieu d'extraction fut parfois obligé comme ce fut le cas lors des attaques des Turcs contre les carrières de l'île noire qui obligèrent à extraire la pierre (d'une qualité apparemment inférieure) à la pointe de Ses Portes ?; les pierres étaient parfois même travaillées hors de l'île. 


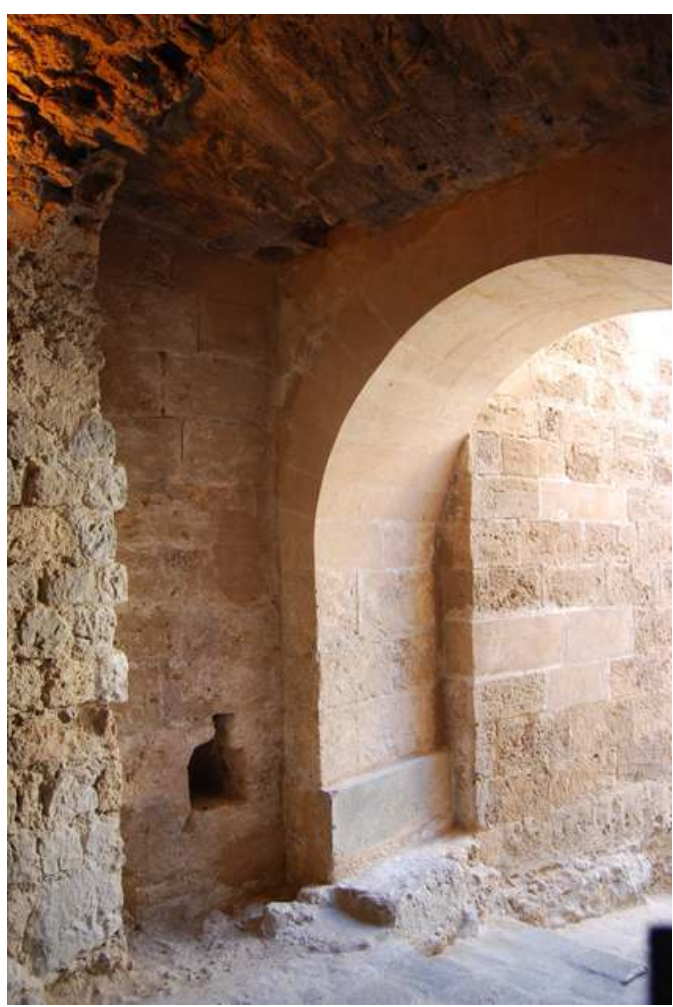

Jambage et extrados de l'arc du Portal Nou une fois retiré le revêtement du XIXe siècle (sur la gauche) où l'on peut observer les retouches successives à la Renaissance grâce aux cavités creusées par les ferrures originelles de la porte et de la barre de fermeture, cachées jusqu'ici.

(c) F. Cobos, maîtrise d'œuvre du plan directeur.

Le premier cas est représenté par la pathologie du Portal Nou (Calvi 1554) où la dégradation de la pierre devait être si importante qu'au XIX ${ }^{e}$ siècle on décida de doubler d'un revêtement l'intérieur de l'arc et de piquer de 12 à 15 centimètres sur la façade extérieure pour y placer un placage, qui lui aussi se dégrada rapidement et fut réparé par la suite à l'aide de plâtrages et de mortiers. L'intervention projetée a consisté à retirer les plâtrages et les placages, à retrouver les proportions et la stéréotomie originelle de l'œuvre de la Renaissance, pierre après pierre, à partir des éléments conservés mais jusque-là dissimulés. 


\section{Figure 21}

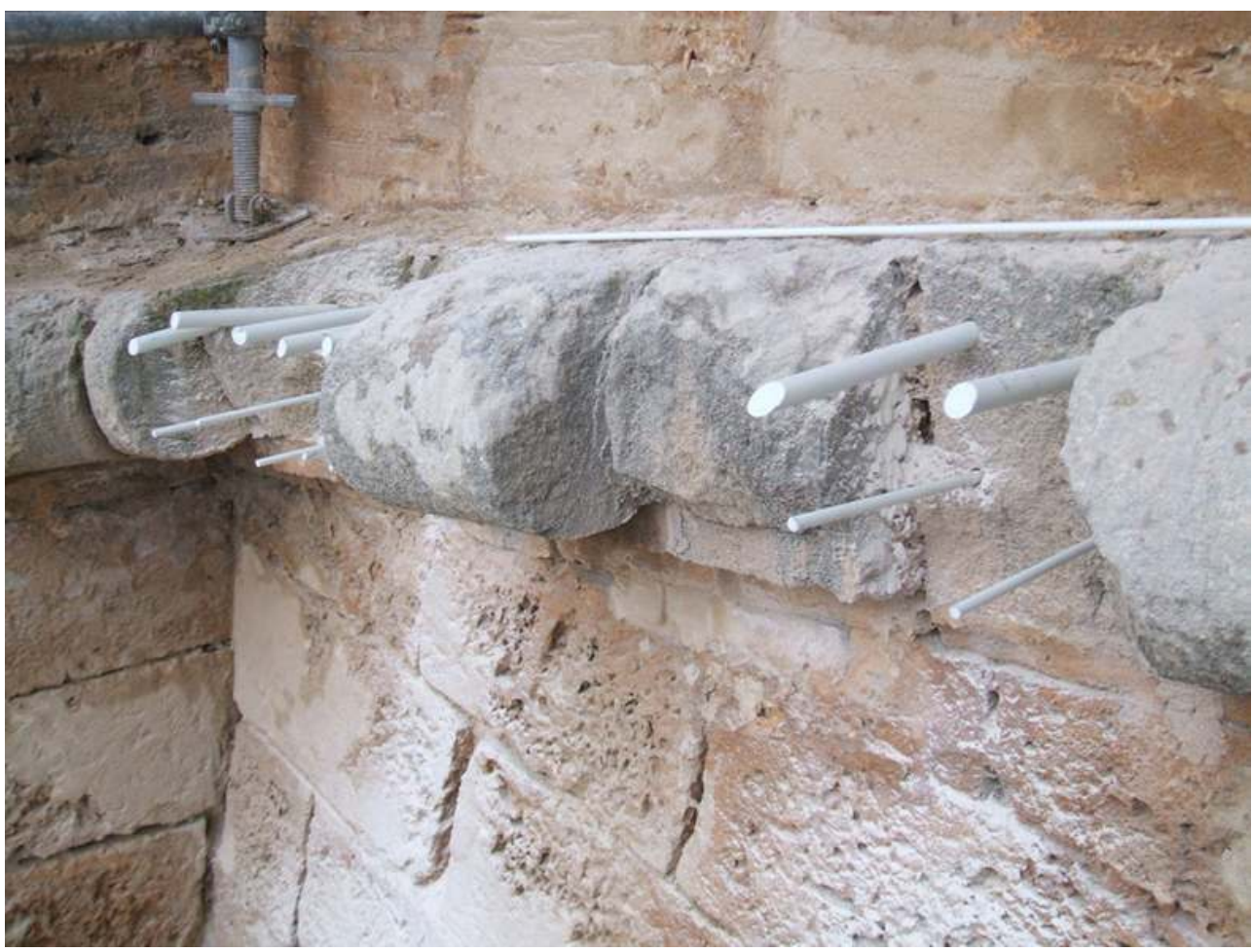

«Le cordon des murailles de la Renaissance souligne le tracé géométrique de la forteresse (comme le mur est taluté, quand la ligne du cordon et la ligne rasante par rapport au sol ne sont pas parallèles, la ligne rasante ne coïncide pas avec le tracé). La dégradation du cordon, outre le fait qu'elle présente un danger pour les passants, laisse à découvert le parement des écoulements. Dans le cas d'lbiza, la taille des pièces et la profondeur de l'enchâssement rendaient impossible leur remplacement par d'autres pièces de pierre. On a donc choisi la réhabilitation à l'aide de mortiers armés spéciaux pour la remise en place ».

(c) F. Cobos, maîtrise d'œuvre du plan directeur. 
Figure 22

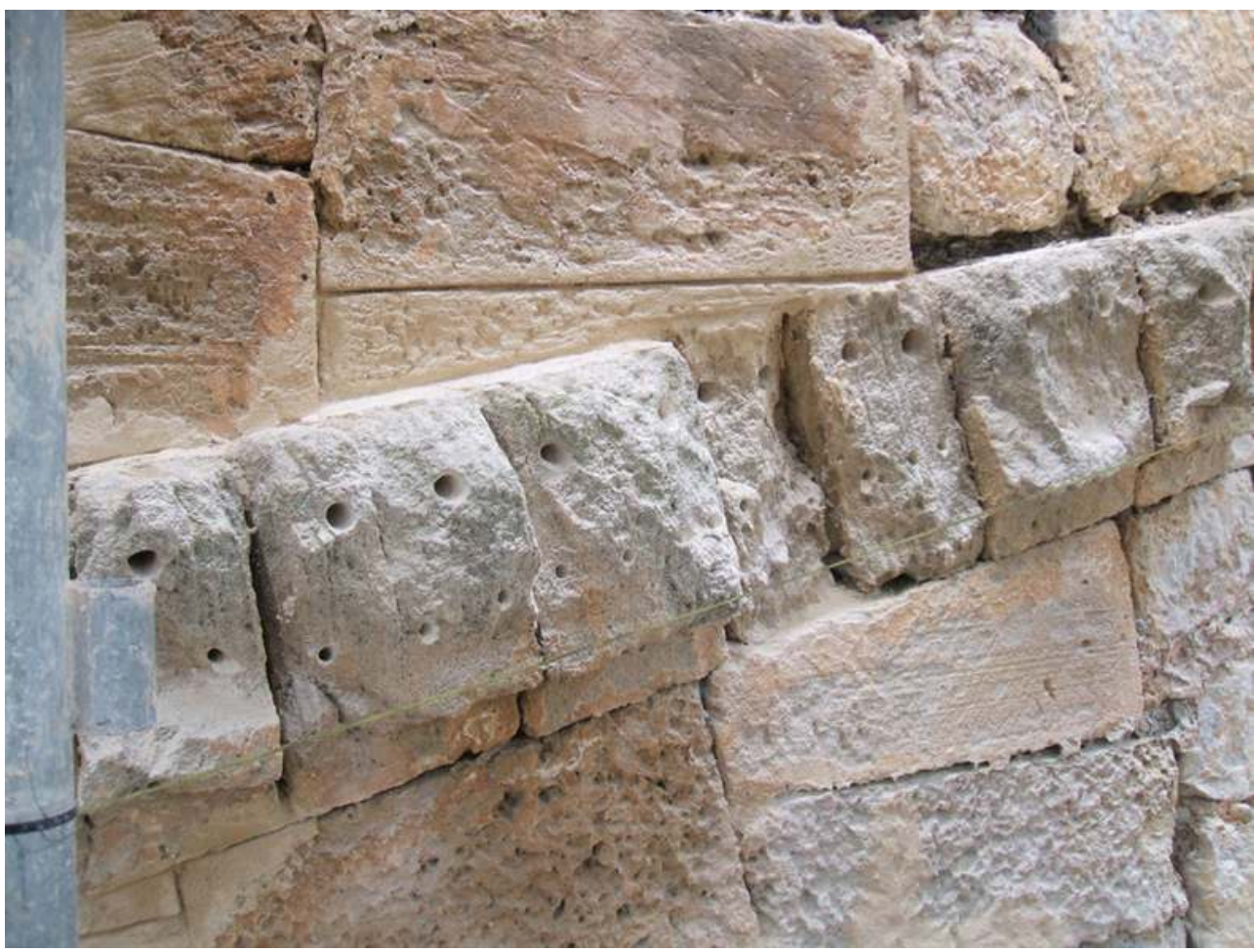

Voir légende figure 21.

Figure 23

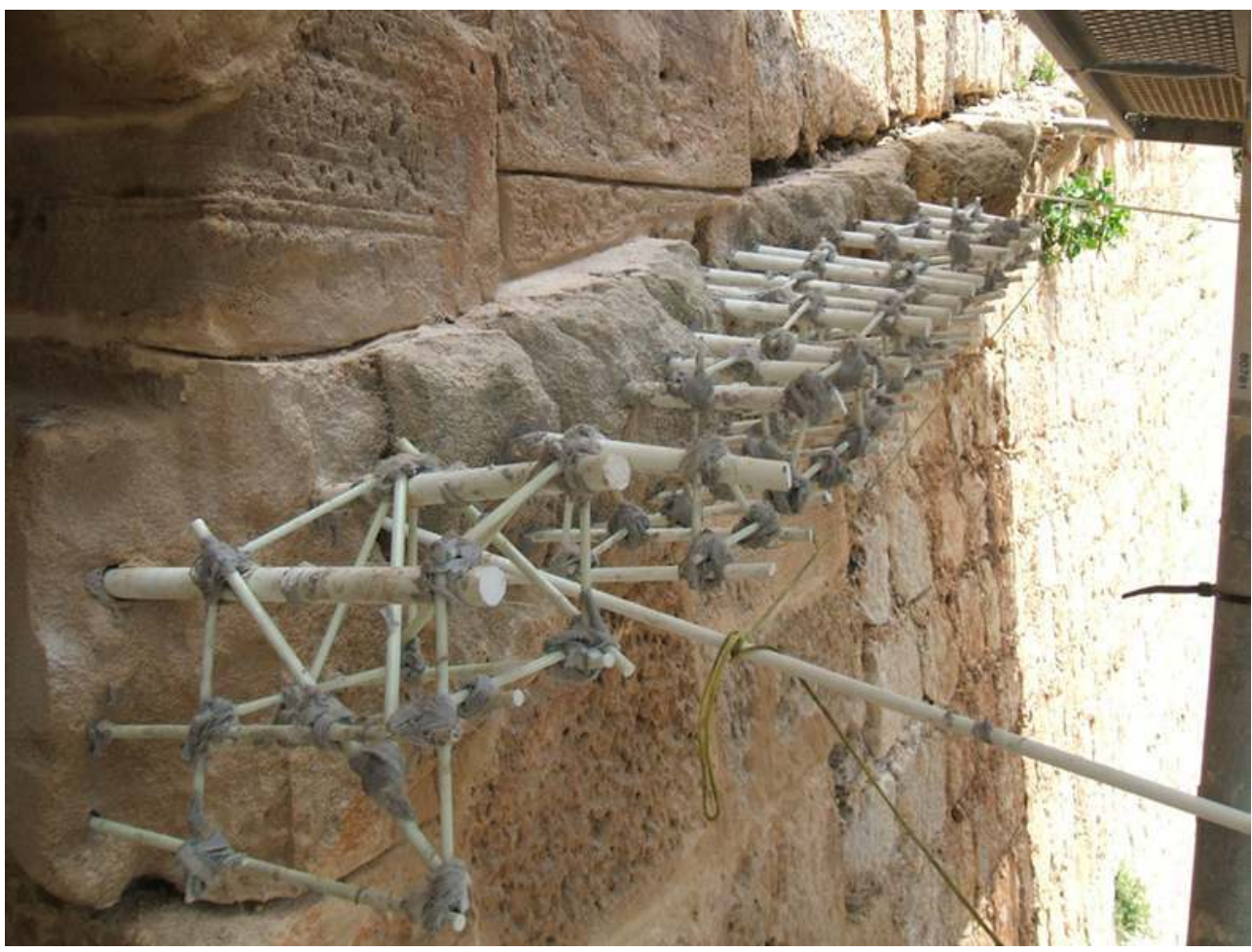

Voir légende figure 21. 
Figure 24

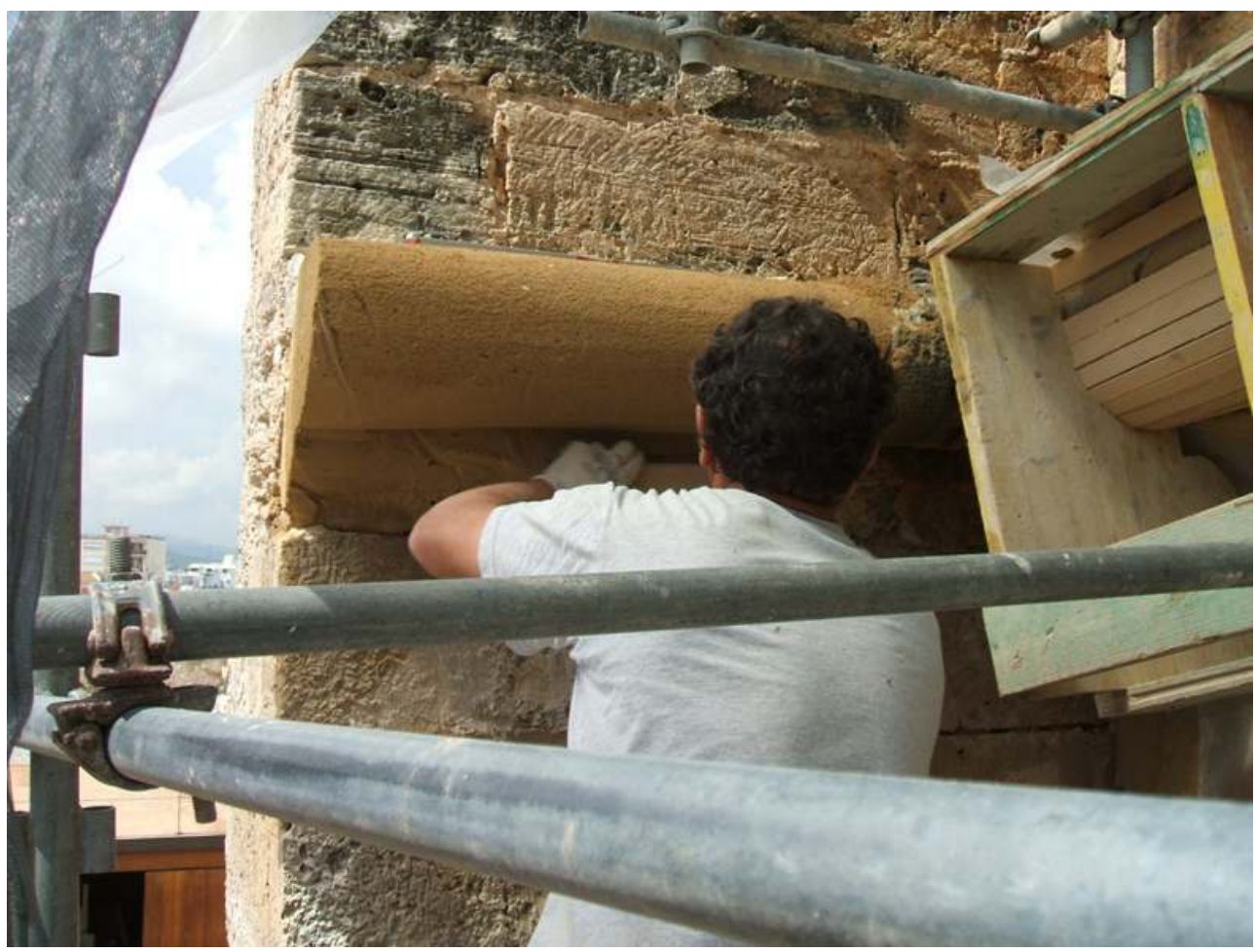

Voir légende figure 21.

\section{Figure 25}

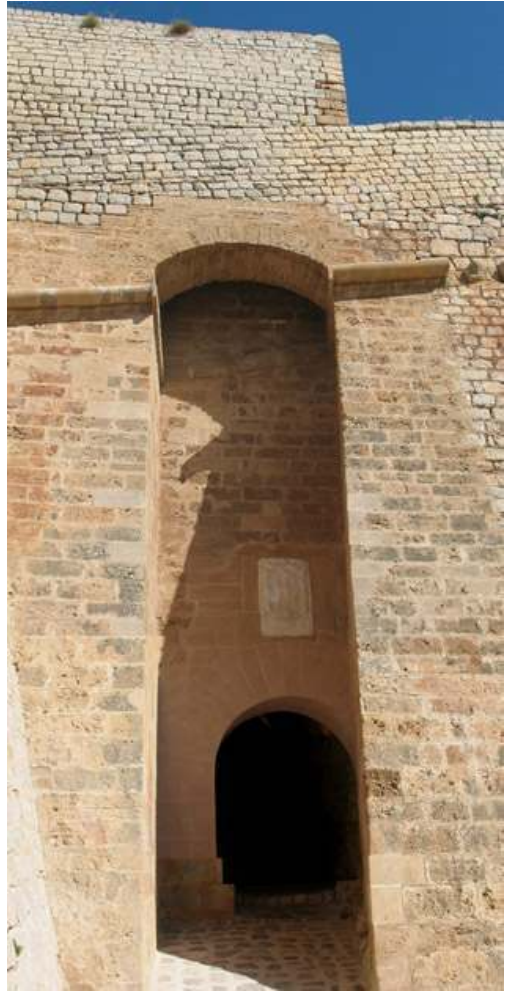

Voir légende figure 21. 
11 La Puerta del Mar, que les habitants d'Ibiza appellent « le portail des planches » (à cause du pont-levis), fut conçue entre 1558 et 1559 pour représenter le pouvoir impérial face aux Turcs. "PHILIPPE ROI CATHOLIQUE INVAINCU DES ESPAGNES ET DES INDES ORIENTALES ET OCCIDENTALES ", peut-on lire sur l'inscription, et cette idée est clairement transmise par le beau tracé bien proportionné et le remploi de sculptures romaines selon un programme iconographique bien précis qui ne s'explique sans doute qu'en admettant qu'elles ont été apportées ex profeso de Cartagena ou de Sagunto et ne sont pas le fruit d'une découverte locale. Le blason, dont la pierre, en provenance de Majorque, est arrivée peut-être déjà travaillée, couronne une porte que la colonisation par des champignons (une pathologie qui curieusement est endémique spécialement dans la cas de la pierre de Majorque) et d'autres colonisations végétales avaient rendue illisible et gravement altérée. L'intervention a impliqué son nettoyage et sa consolidation. Elle a été étendue à la Place d'Armes dans son ensemble et aux parements intérieurs de la porte où les dommages importants étaient dus «seulement " aux pathologies propres à la pierre de Marès d'Ibiza.

Figure 26

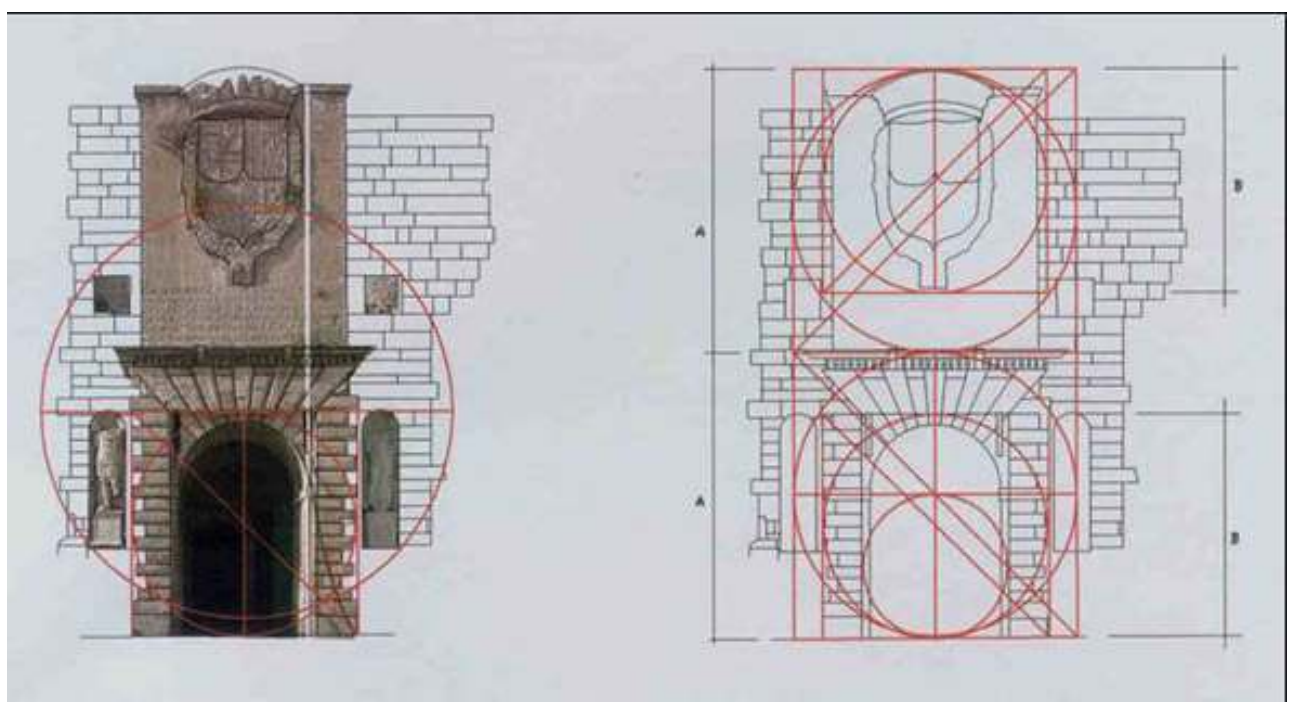

Étude du tracé, des proportions et des pathologies du Portal de las Tablas (« portail des planches »). (c) F. Cobos, cabinet de maîtrise d'œuvre du plan directeur. 


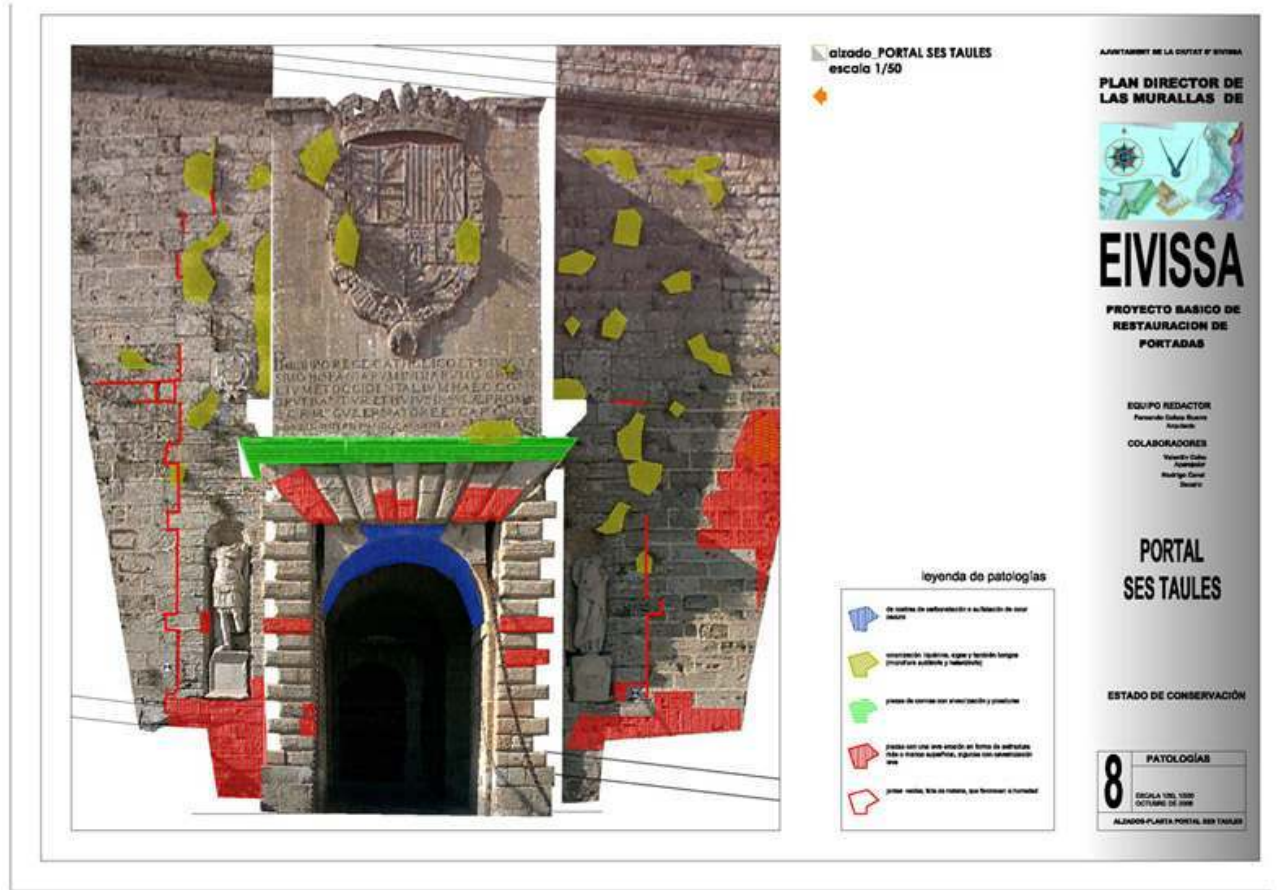

Étude du tracé, des proportions et des pathologies du Portal de las Tablas (« portail des planches »). (c) F. Cobos, cabinet de maîtrise d'œuvre du plan directeur.

Figure 28

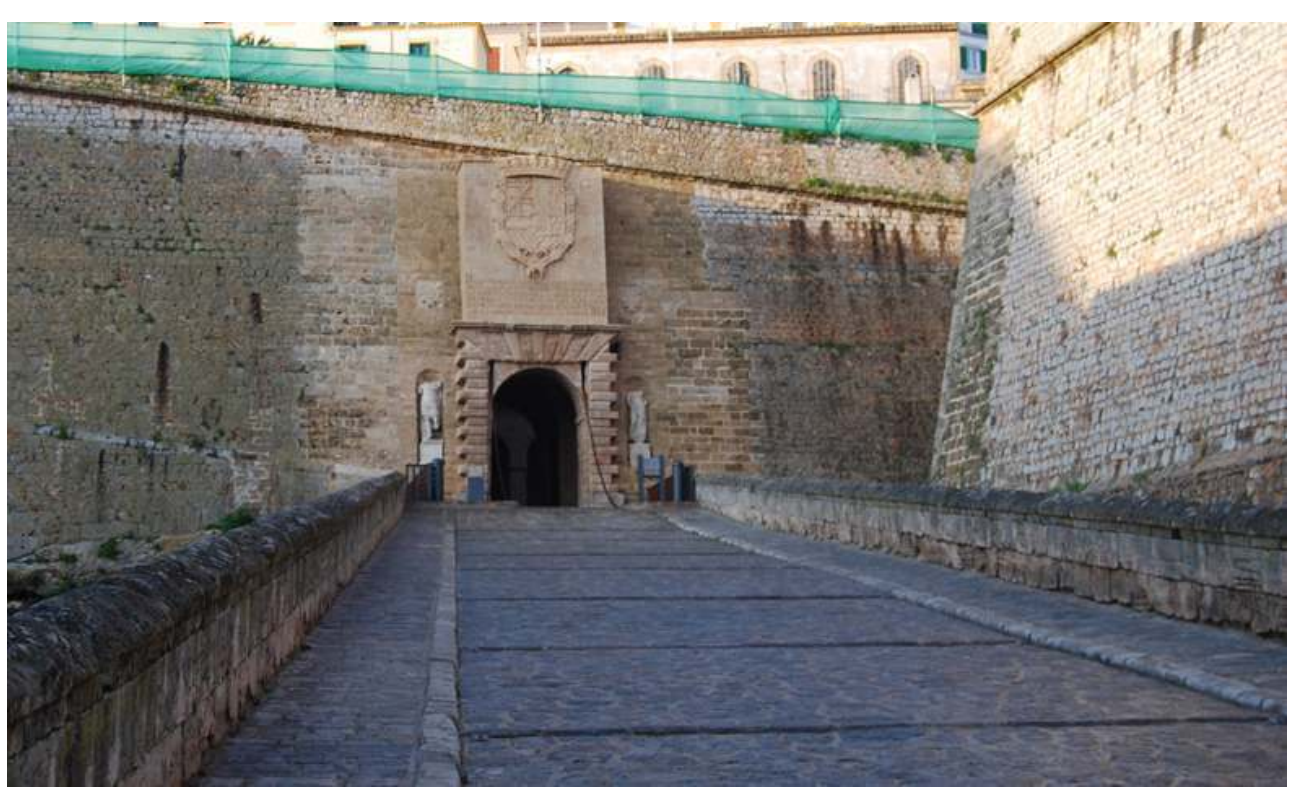

Rampe d'accès au Portal de las Tablas, près du bastion de San Juan. (c) F. Cobos, maîtrise d'œuvre du plan directeur. 


\section{Figure 29}

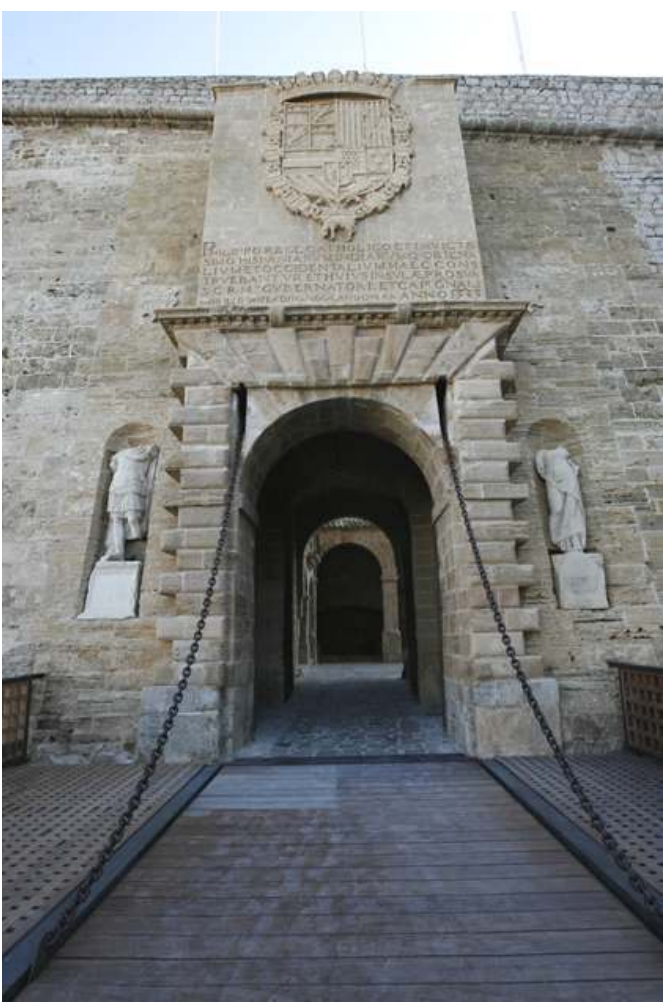

Vue générale de la façade impériale du Portal de las Tablas après sa restauration. (c) F. Cobos, maîtrise d'œuvre du plan directeur.

\section{Figure 30}

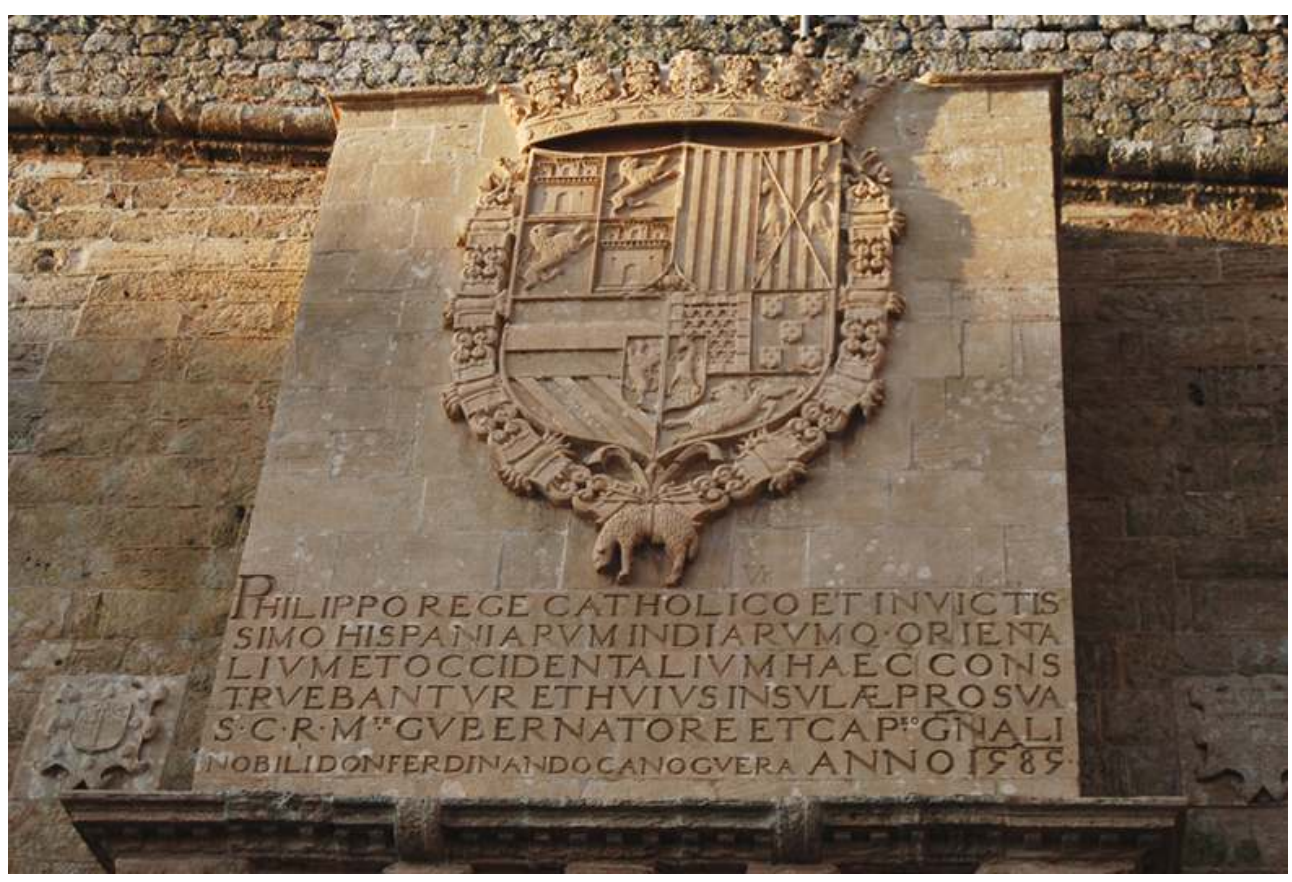

Détail des armoiries de Philippe II, avec leur inscription après la restauration.

(c) F. Cobos, maîtrise d'œuvre du plan directeur 
Fiche technique

- Promoteur : Mairie d'Ibiza

- Financement : Mairie d'Ibiza, Consortium Patrimoine de l'Humanité d'Ibiza et $1 \%$ culturel du Gouvernement espagnol.

- Plan Directeur : Fernando Cobos, architecte et directeur de l'équipe, Manuel Retuerce, archéologue, Mónica Rosselló, restauratrice, Alicia Cámara, historienne. (Autres collaborateurs : les architectes Valentín Cobo, José Luis Fajardo et Mercedes González, l'équipe de topographie de la FGUVA, le géologue Roberto Sánchez et les historiens Pablo de la Fuente, Rocío Casas et Germán Prieto).

- Projets et maîtrise d'œuvre : Fernando Cobos, Cabinet Arquitectura SL et Techniciens Municipaux.

- Patrimoine et Archéologie Municipale : Rosa Gurrea

- Entreprises chargées de l'exécution des travaux :

Refoart SL (Chef de travaux : Gabriel Cárdenas Sarralde. Conservateur-restaurateur : Miquel Vidal Femenies. Directeur général : Raúl Barbieri Fontana).

Atelier École d'Ibiza (Directrice : Ángeles Martín)

Figure 31

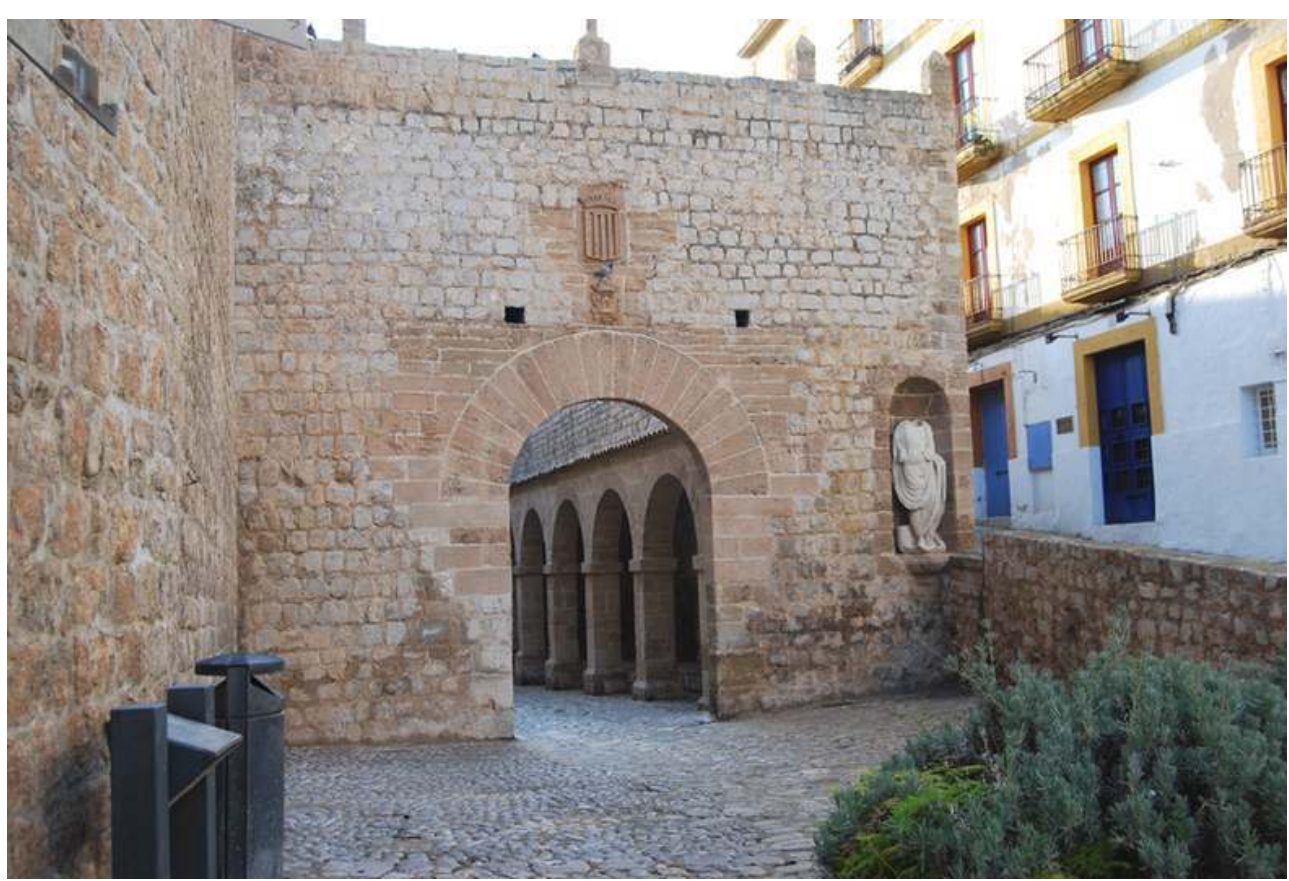

Porte intérieure ou municipale du Portal de las Tablas, galerie de la place d'armes intérieure et Casa de la Guardia (Maison de la Garde).

(c) F. Cobos, maîtrise d'œuvre du plan directeur. 
Figure 32

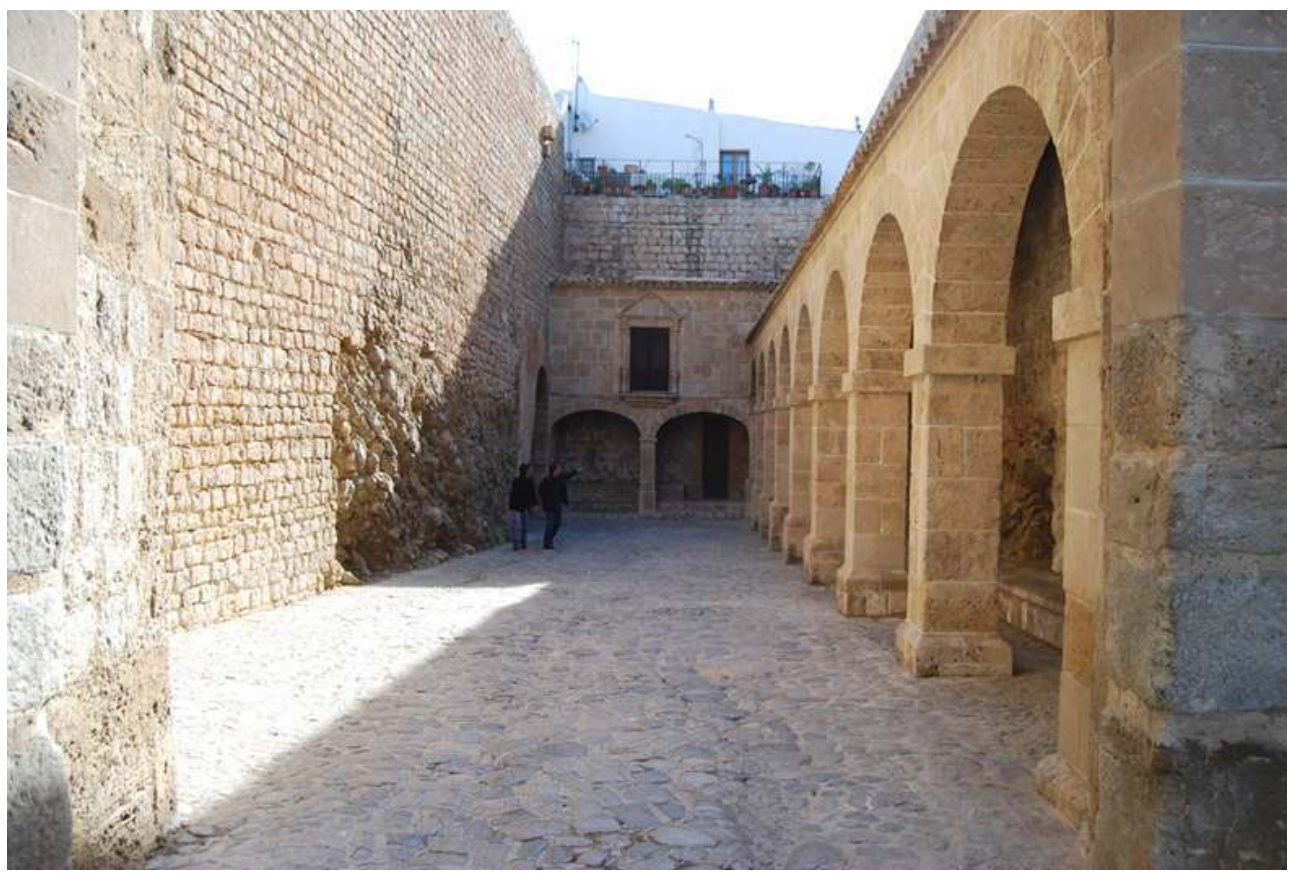

Porte intérieure ou municipale du Portal de las Tablas, galerie de la place d'armes intérieure et Casa de la Guardia (Maison de la Garde).

(c) F. Cobos, maîtrise d'œuvre du plan directeur.

\section{Figure 33}

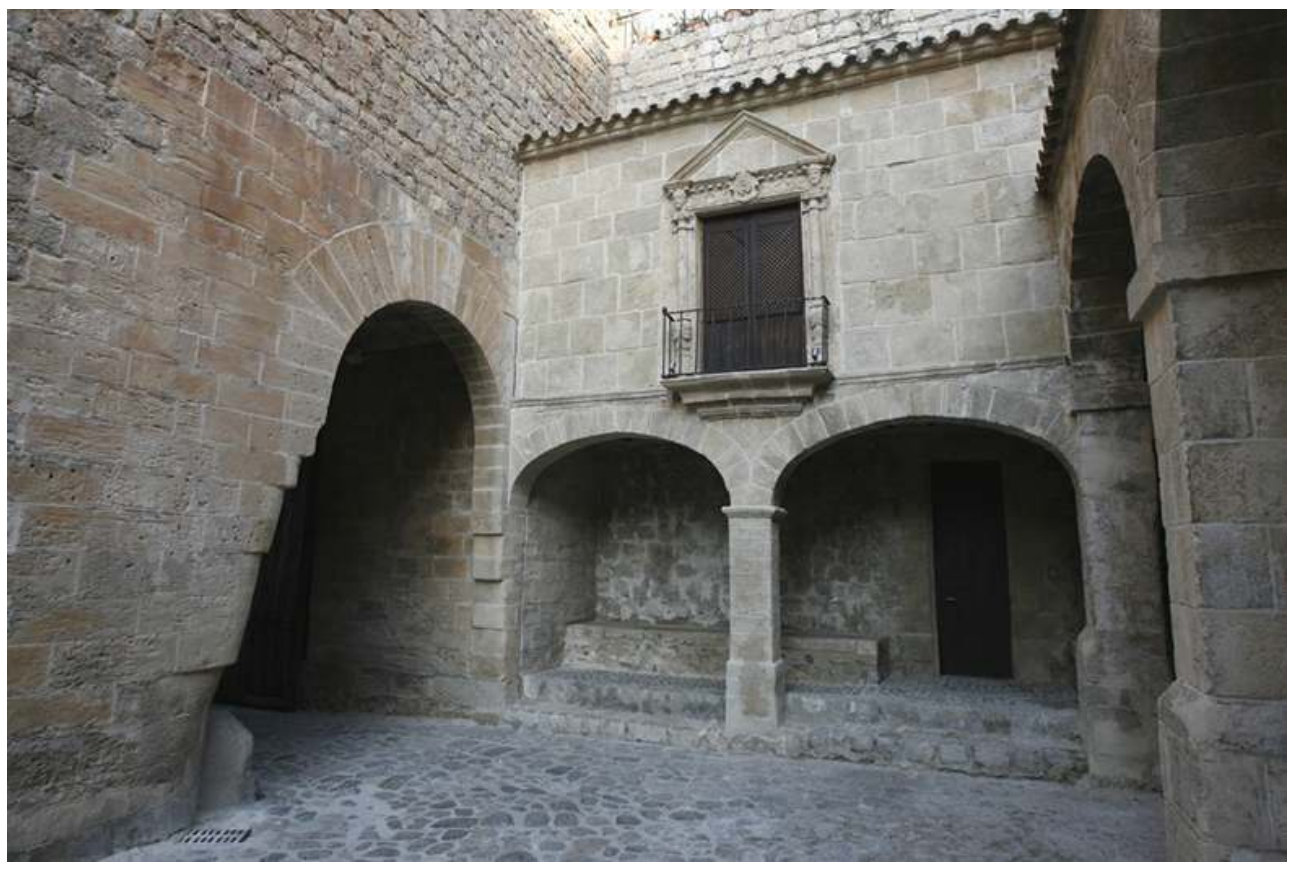

Porte intérieure ou municipale du Portal de las Tablas, galerie de la place d'armes intérieure et Casa de la Guardia (Maison de la Garde).

(c) F. Cobos, maîtrise d'œuvre du plan directeur. 


\section{BIBLIOGRAPHIE}

- Sur la fortification espagnole de l'époque :

COBOS, Fernando. La formulación de los principios de la Fortificación abaluartada: de la Apologia de Escrivá al Tratado de Rojas (1598) in SILVA, M. (ed.) Técnica e ingeniería en España : El renacimiento. Saragosse, 2004.

COBOS, Fernando, CASTRO, Javier de et SÁNCHEZ-GIJÓN, Antonio. Luis Escrivá, su Apología y la Fortificación Imperial. Valence, 2000.

- Sur le Plan Directeur de la Muraille d'Ibiza :

COBOS, Fernando. "El Plan director de las Murallas de Ibiza" in Fortificaciones Americanas y la convención del Patrimonio Mundial. World Heritage Papers $n^{\circ} 19$, UNESCO World Heritage Center. Paris, 2006.

\section{RÉSUMÉS}

Les premiers travaux de restauration programmés dans le cadre du Plan Directeur de la Muraille d'Ibiza récupèrent certains des éléments les plus importants de cet ensemble déclaré Patrimoine Mondial en 1999, suivant les critères du Plan dont l'objectif était de documenter, préserver et réhabiliter le tracé de la Renaissance. Les interventions initiales ont consisté à réhabiliter les casemates et les espaces intérieurs des bastions, à consolider les parapets de la muraille en respectant les différents types existants et à restaurer les deux portes de la Renaissance. De plus, au Portal Nou, on a retiré le revêtement du XIX ${ }^{\mathrm{e}}$ siècle, mettant ainsi au jour l'agencement d'origine et les dimensions de l'arc Renaissance projeté par J.B. Calvi en 1554.

The earliest restoration work carried out the Ibiza city wall, under the terms of an overall restoration plan, saved some of the most important elements of this ensemble, which was declared a UNESCO World Heritage site in 1999. The objectives of the restoration plan were to document, preserve and rehabilitate the Renaissance vestiges. The first steps involved the rehabilitation of the bunkers and the interior spaces of the bastions, the reinforcement of the wall's parapets, all respecting the different types to be found, and the restoration of two Renaissance gates. At the Portal Nou, the nineteenth-century rendering was removed to reveal the original surfaces and the dimensions of the Renaissance arch designed by J. B. Calvi in 1554 .

INDEX

Mots-clés : bastion, casemates, fortification, Ibiza, parapets, Patrimoine mondial, portes, restauration

Keywords : bastions, bunkers, fortifications, gates, Ibiza, parapets, restoration, World Heritage 


\section{AUTEUR}

\section{FERNANDO COBOS-GUERRA}

Architecte expert, patrimoine historique, Valladolid, arquitecto@fernandocobosestudio.com, www.fernandocobosestudio.com 